\title{
Spd-2 Gene Duplication Suggests Cell-Type Specific Assembly Mechanisms of Pericentriolar Material
}

\section{Ryan S. O'Neill, Afeez K. Sodeinde*, Frances C. Welsh*, Carey J. Fagerstrom, Brian J. Galletta and Nasser M. Rusan}

Cell and Developmental Biology Center, National Heart Lung and Blood Institute, National Institutes of Health, Bethesda, MD 20892, USA

*Contributed equally

\section{Correspondence}

oneillrs@nih.gov (R.S.O.), nasser@nih.gov (N.M.R.)

\section{Highlights}

- $\quad$ Spd-2 gene duplication in the Willistoni group is rapidly evolving

- Parent gene $S p d-2 A$ is expressed in somatic cells, whereas the gene duplicate $S p d-2 B$ is expressed in spermatocytes

- $\quad$ Spd-2A organizes pericentriolar material during somatic cell mitosis

- $\quad$ Spd-2B is specialized for pericentriolar material organization during male meiosis 


\section{Abstract}

Centrosomes are multi-protein complexes that function as the major microtubule organizing center (MTOC) for the cell. While centrosomes play tissue-specific MTOC functions, little is known about how particular centrosome proteins are regulated across cell types to achieve these different functions. To investigate this cell type-specific diversity, we searched for gene duplications of centrosome genes in the Drosophila lineage with the aim of identifying centrosome gene duplications where each copy evolved for specialized functions. Through in depth functional analysis of a Spd-2 gene duplication in the Willistoni group, we discovered differences in the regulation of PCM in somatic and male germline cells. The parental gene, $S p d-2 A$, is expressed in somatic cells, where it can function to organize pericentriolar material (PCM) and the mitotic spindle in larval brain neuroblasts. Spd-2A is absent during male meiosis, and even when ectopically expressed in spermatocytes it fails to rescue PCM and spindle organization. In contrast, the new gene duplicate, $S p d-2 B$, is expressed specifically in spermatocytes. During male meiosis, Spd-2B localizes to centrosomes, organizes PCM and spindles, and is sufficient for proper male fertility. Experiments using chimeric transgenes reveal that differences in the C-terminal tails of Spd-2A and Spd-2B are responsible for these functional changes. Thus, $S p d-2 A$ and $S p d-2 B$ have evolved complementary functions by specializing for distinct subsets of cells. Together, our results demonstrate that somatic cells and male germline cells have fundamentally different requirements for PCM, suggesting that PCM proteins such as Spd-2 is differentially regulated across cell types to satisfy distinct requirements. 


\section{Introduction}

Centrosomes are complex organelles made up of many proteins, which together serve as the primary microtubule organizing centers (MTOCs) in most eukaryotic cells (Conduit et al., 2015; Vasquez-Limeta and Loncarek, 2021), ensuring proper spindle formation and chromosome segregation during cell division (Lerit and Poulton, 2016). The structure of the centrosome includes the core centrioles surrounded by a cloud of pericentriolar material (PCM), which anchors $\gamma$-Tubulin and nucleates microtubules (Conduit et al., 2015; Vasquez-Limeta and Loncarek, 2021). Although centrosomes function as MTOCs across many different cell types in an organism, the specific requirements for MTOC activity in a given cell type may be different as a result of cell type-specific characteristics such as cell size, cell cycle speed, or whether the cell undergoes mitotic or meiotic division (Greenan et al., 2010; Kwon and Scholey, 2004; Ohkura, 2015; Rieckhoff et al., 2019).

Relatively little is known about how centrosome proteins achieve cell type-specific differences in their functions. One way to accomplish cell type-specific functions is through alternative splicing. For example, in Drosophila melanogaster the centrosome gene Cnn encodes multiple splicing isoforms (Chen et al., 2017). Whereas most isoforms include a centrosome-targeting domain, one isoform instead includes a mitochondria-targeting domain which transforms the mitochondria into a MTOC during spermatogenesis. A second solution is through cell type-specific differences in binding partners. For example, in $D$. melanogaster PLP requires its binding partner calmodulin for its role the basal body of neurons, while this interaction is dispensable for its role at the basal body of sperm (Galletta et al., 2014). Additionally, post-translational modifications such as phosphorylation or ubiquitination could alter centrosome protein function across cell types.

Another possible solution to modify centrosome protein functions is through gene duplication (Ohno, 1970; Taylor and Raes, 2004). For a single copy gene, mutations that enhances protein function in one cell type may have a detrimental effect on its function in other cell types, thus limiting the potential for single copy genes to achieve optimal function across all cell types. Gene duplication can bypass this limitation by allowing each duplicate to accumulate mutations independently and become specialized for a subset of functions (Des Marais and Rausher, 2008; Hughes, 1994). Some centrosome genes appear to have evolved in this way. For example, a gene duplication of CEP135 in vertebrates gave rise to TSGA10, which has testis-specific expression and appears to be critical for the formation of sperm head-tail attachment (Modarressi et al., 2001; Sha et al., 2018). We reasoned that by identifying and studying centrosome gene duplications that evolved towards cell type-specific specialization, we could gain insight into the nature of cell type-specific differences in MTOC activity, how centrosome protein functions change across cell types, and the mechanisms underlying cell type-specific centrosome protein regulation.

Here, we present our work on a duplication of the Spd-2 gene in Drosophila willistoni. In Caenorhabditis elegans and humans, SPD-2/CEP192 are required for both PCM recruitment and centriole duplication (Gomez-Ferreria and Sharp, 2008; Kemp et al., 2004; Pelletier et al., 2004), whereas D. melanogaster Spd-2 is required for PCM recruitment but not for centriole duplication (Dix and Raff, 2007; Giansanti et 
al., 2008). Spd-2 localizes to the centrosomes where it is required for proper PCM organization in both larval brain neuroblast mitosis and male spermatocyte meiosis (Dix and Raff, 2007; Giansanti et al., 2008). Spd-2 is initially recruited to the centriole wall by the bridge zone protein Asl (Conduit et al., 2014). Once recruited to the centrosome, Spd-2 in turn recruits another PCM protein Cnn, and together they organize a stable PCM for $y$-Tubulin recruitment and microtubule nucleation (Conduit et al., 2010; Conduit et al., 2014; Goshima et al., 2007). However, many questions remain as to how Spd-2 is regulated, and it is unknown whether Spd-2 functions differently in the contexts of mitosis versus meiosis.

Our investigation of $D$. willistoni Spd-2 gene duplicates reveals that the parental gene, $S p d-2 A$, maintained its ability to organize PCM during mitosis but lost the ability to organize PCM during meiosis. In contrast, the rapidly evolving new gene copy, $S p d-2 B$, is specifically expressed in the testes where it functions to organize meiotic PCM. Experiments using chimeric transgenes reveal that the amino acid changes responsible for differences in meiotic PCM assembly function occurred in the C-terminal tail of the Spd-2 duplicates, suggesting that the C-terminal region of Spd-2 is a target of regulation to achieve differences in PCM function between mitotic and meiotic cell division. 


\section{Results}

\section{Screen for Centrosome Gene Duplications in the Drosophila Lineage}

To identify duplications of centrosome genes in the Drosophila lineage, we used BLAST to screen $13 D$. melanogaster centrosome proteins (Figure 1A) against the annotated protein sequence databases of 35 Drosophila species (Supplemental File 1). Of the 13 genes, five have undergone duplication at least once (Figure 1B). Sas-4, which encodes a core centriolar protein, has a nearly identical tandem duplication in Drosophila yakuba. Asl, Cnn, and Cp110 were all duplicated multiple times. Interestingly, both Asl duplications (AsI-B and AsI-C) were subsequently re-duplicated in different lineages (Figure 1B, Asl). The Cnn duplication in Drosophila miranda was nearly identical to the parent gene, whereas a second duplication found in several species was a highly divergent partial duplication containing only a small portion of the N-terminal protein coding sequence (Figure 1B, Cnn). Finally, Spd-2 was duplicated in both $D$. melanogaster and $D$. willistoni. In $D$. melanogaster, we found the previously identified chimeric pseudogene CR18217, which contains an N-terminal fragment of Spd-2 along with the NUDIX domain from CG4098 (Rogers and Hartl, 2012). In D. willistoni, the new copy of Spd-2 encoded a nearly full length protein that showed substantial divergence from its parent gene. We chose the $S p d-2$ gene duplication for detailed analysis, and herein refer to the $D$. willistoni parent gene as $S p d-2 A$, the $D$. willistoni new gene duplicate as $S p d-2 B$, and the $D$. melanogaster single copy gene as Spd-2.

\section{Spd-2B is a Rapidly Evolving Duplication of Spd-2A}

We obtained the genome sequence upstream and downstream of $S p d-2 A$ and used BLAST to determine the corresponding regions in the $D$. melanogaster genome, revealing the same upstream and downstream genes for both $S p d-2 A$ and $S p d-2$ and thus confirming their orthology (Figure $2 A$ ). We used the same strategy to identify the locus of $S p d-2 B$, which was between the bug and Mlf genes in $D$. willistoni; in D. melanogaster, bug and MIf are directly adjacent on Chromosome 2 (Figure 2A).

To determine whether $S p d-2 B$ is a $D$. willistoni-specific duplication we used PCR and sequencing to screen for $S p d-2 A$ and $S p d-2 B$ in six species of the Willistoni group. PCR screening revealed that both $S p d-2 A$ and $S p d-2 B$ were present in all six species (Figure $2 B$, Figure $S 1 A$ ), whereas $S p d-2 B$ was not found in the outgroup species $D$. virilis. Thus, the duplication that gave rise to $S p d-2 B$ likely occurred in the ancestor of the Willistoni group, between $\sim 22-50$ million years ago (Russo et al., 2013), and was subsequently retained in all daughter species.

We next assessed the sequence conservation of $S p d-2 A$ compared to $S p d-2 B$. We generated a codon alignment of $S p d-2$ homologs from seven species. This codon alignment revealed that $S p d-2 B$ is evolving at a significantly faster rate compared to Spd-2A (Figure $2 \mathrm{C}$ ). To assess conservation of protein sequence and structure, we compare Spd-2A and Spd-2B to Spd-2 protein sequences (Figure 2D). Compared to $S p d 2, S p d-2 B$ is less conserved than Spd-2A and contains over twice the number of amino acid indels. The C-terminal halves of Spd-2A and Spd-2B are more strongly conserved compared to their $\mathrm{N}$-terminal halves. However, Spd-2B is missing the C-terminal 119 amino acid tail of Spd-2A and Spd-2. Together, 
these results indicate that $S p d-2 B$ is evolving more rapidly than $S p d-2 A$, suggesting functional divergence or specialization for $S p d-2 B$.

\section{Spd-2A, but not Spd-2B, organizes PCM and the Mitotic Spindle in Larval Brain Neuroblasts}

To explore the functions of $\mathrm{Spd}-2 \mathrm{~A}$ and $\mathrm{Spd}-2 \mathrm{~B}$, we generated $D$. melanogaster animals expressing GFPtagged transgenes of $S p d-2 A, S p d-2 B$, and $S p d-2$. To preserve major regulatory elements and reflect native expression patterns, each transgene included their complete native upstream and downstream intergenic DNA sequences and were inserted into the same insulated attP landing site in the $D$. melanogaster genome (Figure S2A). Whereas both native promoter mel(p)-Spd-2::GFP and wil(p)-Spd2A::GFP were expressed in the larval brain neuroblasts (Figure 3A, B), wil(p)-Spd-2B::GFP was not detectable (Figure 3C). Similarly, mel(p)-Spd-2::GFP and wil(p)-Spd-2A::GFP, but not wil(p)-Spd-2B::GFP, were expressed in larval wing discs (Figure S2B-D). Thus, Spd-2 and Spd-2A are expressed in somatic tissues, whereas Spd-2B is absent.

Larval brain neuroblasts in metaphase have well organized mitotic spindle poles and radial astral microtubules (Figure 3D). Similar to previous reports, we found that spd-2- neuroblast metaphase spindles were abnormally barrel shaped with unfocused poles that also lack astral microtubules (Figure 3E; Giansanti et al., 2008). Expressing mel(p)-Spd-2::GFP or wil(p)-Spd-2A::GFP transgenes in the spd-2mutant background rescued these abnormal metaphase spindle defects (Figure 3F, G). However, wil(p)$S p d-2 B:: G F P$ transgene failed to rescue the $s p d-2^{-}$spindle phenotypes (Figure $3 \mathrm{H}$ ), consistent with the lack of GFP expression in larval brain neuroblasts. Ectopically expressing a UAS-Spd-2B::GFP transgene using actin-GAL4 in the spd-2- background fully rescued the mutant spindle defects (Figure 31 ). This indicates that Spd-2B protein is capable of performing Spd-2 or Spd-2A function in the brain, but Spd-2B is transcriptionally inactive in the brain.

We next wondered if Spd-2A could fully rescue centrosome assembly in somatic cells. During neuroblast mitosis, Spd-2 is known to recruit and stabilize Cnn to organize and expand the PCM (Conduit et al., 2010; Conduit et al., 2014; Goshima et al., 2007). We found that, similar to previous reports, spd-2neuroblasts are often missing centrosomes and fail to robustly recruit $\psi$-Tubulin and $\mathrm{Cnn}$ as compared to controls (Figure 4A, B, G-I; Conduit et al., 2007; Giansanti et al., 2008; Goshima et al., 2007). Both mel(p)-Spd-2::GFP and wil(p)-Spd-2A::GFP localize to centrosomes and rescue spd-2 recruitment of both $\gamma$-Tubulin and Cnn (Figure 4C, D). As expected, wil(p)-Spd-2B::GFP is undetectable in larval brain neuroblasts, and fails to rescue spd-2 centrosome numbers, $\gamma$-Tubulin recruitment or $\mathrm{Cnn}$ recruitment (Figure 4E). However, consistent with mitotic spindle rescue, UAS-Spd-2B::GFP ectopic expression via actin-GAL4 localizes to centrosomes and fully rescues $s p d-2^{-}$centrosome number and recruitment of $\mathrm{Y}^{-}$ Tubulin and $\mathrm{Cnn}$ (Figure 4F-I). Together these results show that, although Spd-2B is not normally expressed in larval brain cells, the encoded protein is fully capable of organizing the mitotic spindle and PCM via Cnn and $y$-Tubulin recruitment.

\section{Spd-2A and Spd-2B have complementary centriolar localizations during spermatogenesis}


In addition to its roles in larval brain neuroblasts, Spd-2 has also been studied for its role in male meiosis. First, we characterized the expression patterns of native promoter transgenes by looking at centrosomal localization of Spd-2::GFP at various stages during spermatogenesis. These stages included the germline stem cells and spermatogonia, which undergo mitotic divisions (Figure 5A), mature premeiotic spermatocytes (Figure 5B), and spermatocytes, which undergo meiosis (Figure 5C). During mitotic stages, both mel( $p$ )-Spd-2::GFP and wil(p)-Spd-2A::GFP localized to centrosomes and expanded into PCM, whereas wil( $p$ )-Spd-2B::GFP was barely detectable (Figure $5 \mathrm{~A})$. In pre-meiotic mature spermatocytes this pattern is reversed, with mel(p)-Spd-2::GFP and wil(p)-Spd-2B::GFP localized to centrosomes, whereas wil( $p$ )-Spd-2A::GFP was now barely detectable (Figure $5 B$ ). Finally, this pattern continued through the meiotic divisions, with mel(p)-Spd-2::GFP and wil(p)-Spd-2B::GFP localized to centrosomes and expanding into PCM, and wil(p)-Spd-2A::GFP was still barely detectable (Figure $5 C$ ). The cytoplasmic level of Spd-2A::GFP was equal in mitotic and meiotic stages (Figure 5D), indicating that the reduction in centrosomal Spd-2A::GFP was not due to total cellular protein degradation but rather a delocalization of Spd-2A::GFP off of the centrosome. The increase of Spd-2B::GFP on the centrosome and PCM in later stages was accompanied by a global cytoplasmic increase of Spd-2B::GFP (Figure 5D), suggesting transcriptional or translational control of Spd-2B::GFP. Nonetheless, the levels of cytoplasmic Spd-2B::GFP were no greater than Spd-2A::GFP in meiotic spermatocytes, yet only Spd-2B::GFP is loaded onto the centriole and expanded into the PCM, strongly suggesting that Spd2A and Spd2B protein are differentially regulated, possibly via unique phosphorylation sites. Together, these results show that Spd-2A and Spd-2B have complementary patterns of centrosomal localization such that a combination of both are required to recapitulate the full localization of Spd-2 throughout all stages of spermatogenesis.

\section{Spd-2B is Specialized for PCM Organization During Male Meiosis}

Next, we investigated the PCM recruitment functions of Spd-2A and Spd-2B. Wild type spermatocytes recruit $\gamma$-Tubulin into the PCM during meiosis I (Figure 6A), whereas spd-2 spermatocytes only recruit a small amount of $\gamma$-Tubulin that fails to spread into PCM (Figure 6B). mel(p)-Spd-2::GFP rescues spd-2 $\gamma^{-}$ Tubulin recruitment, causing a similar sized PCM, albeit with higher than wild type $\gamma$-Tubulin levels (Figure 6C). As expected based on lack of localization, wil(p)-Spd-2A::GFP fails to rescue spd-2 $\gamma$-Tubulin recruitment (Figure 6D). In contrast, wil(p)-Spd-2B::GFP rescues $s p d-2-\gamma$-Tubulin recruitment, causing a similar sized centrosome with similar $\gamma$-Tubulin levels compared to controls, but qualitatively less than mel(p)-Spd-2::GFP (Figure 6E). We also investigated the meiotic spindle organizing functions of Spd-2A and Spd-2B. In meiosis I, controls organize a proper meiotic spindle with robust asters (Figure 6F), whereas spd-2 spermatocyte centrosomes are abnormal, recruiting very weak or no astral microtubules, and are frequently detached from the poles (Figure 6G, H). mel( $p$ )-Spd-2::GFP fully rescues spd-2 meiotic spindle defects (Figure 6I). As expected, wil(p)-Spd-2A::GFP fails to rescue spd-2 meiotic spindle defects (Figure 6J) whereas wil(p)-Spd-2B::GFP fully rescues (Figure 6K). Thus, consistent with localization patterns (Figure 5), Spd-2B, but not Spd2A, controls PCM assembly and spindle organizing functions during male meiosis. 
To further test the functions of Spd-2A and Spd-2B in spermatogenesis, we tested whether they were sufficient to rescue $s p d-2^{-}$male sterility. We allowed individual males to mate with two virgin females over the course of four days, and then counted the total number of offspring (Figure 7A). Wild type males produced an average 127 offspring. As previously reported, spd-2- males were completely sterile, producing zero offspring (Dix and Raff, 2007; Giansanti et al., 2008). mel(p)-Spd-2::GFP rescued spd-2male sterility, with an average 95 offspring per male. As expected based on localization and ability to rescue $y$-Tubulin recruitment, will(p)-Spd-2A::GFP completely failed to rescue spd-2- male sterility. In contrast, wil(p)-Spd-2B::GFP rescued spd-2- male sterility similar to mel(p)-Spd-2::GFP, with an average 99 offspring per male. Together, along with $\psi$-Tubulin rescue these results show that $S p d-2 B$ is specialized for spermatogenesis.

Next, we ectopically overexpressed UAS transgenes in wild type spermatocytes using topi-GAL4 to increase protein levels during meiosis and potentially force centrosomal localization. Spd-2::GFP had robust centriolar localization in spermatocytes which expanded into the PCM during meiosis (Figure 8A). Spd-2A::GFP was weakly localized to centrioles and meiotic PCM of spermatocytes (Figure 8B). In contrast, Spd-2B::GFP had robust centriolar localization and expansion into meiotic PCM (Figure 8C). We also tested whether topi-GAL4-driven UAS transgenes could rescue spd-2- meiotic spindle organization. topi-GAL4 driving UAS-Spd-2::GFP fully rescues spindles (Figure $8 D$, E). In contrast, topi-GAL4 driving UAS-Spd-2A::GFP fails to rescue spindles (Figure 8F). Finally, topi-GAL4 driving UAS-Spd-2B::GFP fully rescues spindles (Figure $8 \mathrm{G}$ ). Thus, even when ectopically overexpressed in spermatocytes Spd-2A localizes to meiotic centrosomes at low levels, does not expand into the PCM, and does not organize meiotic spindles, suggesting that $S p d-2 A$ has accumulated coding sequence mutations through the course of its evolution that led to loss of ancestral Spd-2 meiotic PCM functions.

\section{Spd-2A C-terminal Tail Prevents Meiotic Spindle Organizing Function}

To determine the specific amino acid changes responsible for the differences in Spd-2A and Spd-2B function we used chimeric transgenes as an initial attempt to map the meiotic function to a specific protein region. First, we combined the $\mathrm{N}$-terminal half of $\mathrm{Spd}-2 \mathrm{~A}$ with the $\mathrm{C}$-terminal half of $\mathrm{Spd}-2 \mathrm{~B}$, and vice versa, to make UAS-Spd-2A-B ${ }^{\text {chimera }}$ and UAS-Spd-2B-A $A^{\text {chimera }}$, respectively. topi-GAL4 driving UAS-Spd$2 A-B^{\text {chimera }}:: G F P$ fully rescues $s p d-2^{-}$meiotic spindle defects (Figure 9A, B), whereas UAS-Spd-2B-

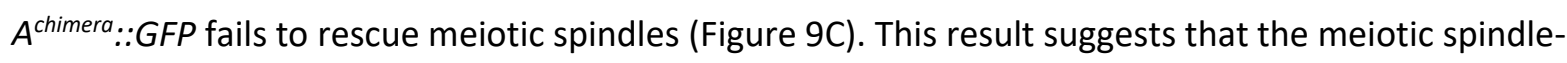
organizing function can be attributed to the $\mathrm{C}$-terminal half of $\mathrm{Spd}-2 \mathrm{~B}$. The most conspicuous difference between Spd-2A and Spd-2B is the lack of the C-terminal-most $\sim 119$ amino acids (the tail) in Spd-2B (Figure 2D). We therefore generated additional transgenes where we removed the tail from Spd-2A

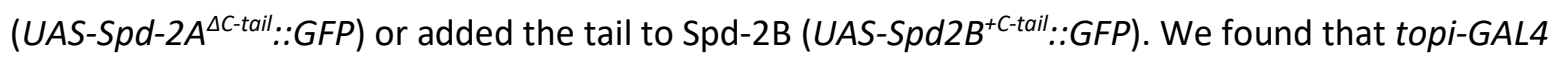

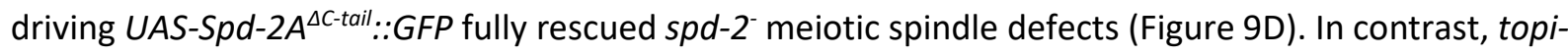
GAL4 driving UAS-Spd2 $B^{+C-\text { tail }:: G F P ~ f a i l s ~ t o ~ r e s c u e ~} s p d-2^{-}$meiotic spindle defects (Figure 9E). Thus, the presence of the C-terminal tail of Spd-2A is responsible for its inability to organize meiotic PCM and spindles. 


\section{Discussion}

In this study, we sought to gain insight into the cell type-specific functions of centrosome proteins using an evolutionary approach. In D. melanogaster, Spd-2 functions in both mitotic and meiotic divisions (Dix and Raff, 2007; Giansanti et al., 2008). The most counter-intuitive result from our study is that although Spd-2A is the more highly conserved paralog and contains all protein regions found in Spd-2, it has lost the ability to function in PCM recruitment in meiotic cells (Figure 6D). Instead, the less well-conserved Spd-2B is capable of expanding into the PCM in meiotic cells (Figure 6E), and in mitotic cells when ectopically expressed (Figure 4F). We also found that the C-terminal 119 amino acid tail of Spd-2A, which is absent from Spd-2B, is responsible for preventing meiotic function (Figure 9D, E), explaining why even higher, ectopic expression of $\mathrm{Spd}-2 \mathrm{~A}$ is still unable to rescue meiotic function (Figure $8 \mathrm{~B}$ ). Interestingly, removing the tail from Spd-2A rescues meiotic function, indicating that the PCM recruitment capability of Spd-2A is not lost and must be located in another region of the protein. These results suggest a two-step process for ancestral (and D. melanogaster) Spd-2 in driving PCM assembly: Step 1) Spd-2 priming via tail autoinhibition relief; Step 2) Spd-2 activation for PCM recruitment (Figure 10A).

Previous work on 'Step 2' in multiple systems showed that Spd-2/SPD-2/Cep192 are activated via phosphorylated during mitosis, and that this phosphorylation is required to efficiently recruit Polo/Plk1 and expand the mitotic PCM (Alvarez-Rodrigo et al., 2019; Decker et al., 2011; Meng et al., 2015). Our work does not shed light on 'Step 2' except to show that it does not require the C-terminal tail. Instead, our work has identified 'Step 1', a priming step that provides an additional level of Spd-2 regulation. Our results provide at least two plausible explanations for autoinhibition relief: A) autoinhibition by the tail is male meiosis-specific. In this case, Spd-2 priming is only necessary in meiosis, while in mitosis 'Step 2' is sufficient for PCM regulation. B) A second, more favorable explanation is that there are distinct cell type-specific (mitotic and meiotic) mechanisms of autoinhibition relief to prime Spd-2 (Figure 10A). The mechanism of relief could involve a cell type specific binding partner (a protein activator), or a cell type specific post-translational modification at a distinct mitotic residue(s) and meiotic residue(s) within Spd2 (Figure 10A, priming sites). Data from the literature supports this two step model and provides many hypotheses that need future testing. For example, although evidence from $C$. elegans indicates that SPD2 exists in a monomeric state in the cytoplasm, intramolecular Spd-2 interactions have been reported by several groups via yeast two-hybrid (Conduit et al., 2014; Galletta et al., 2016a). Thus, one possibility is that the priming step represents a switch from a monomeric state during interphase to an oligomeric state at the centrosome during mitosis, which somehow involves tail disengagement. Future work should aim to better understand the nature of this putative mechanism and why it appears to function differently across cell types.

Spd-2B likely arose via a small-scale, dispersed DNA-based duplication mechanism (Ezawa et al., 2011) as evidenced by the presence of introns and its location on a different chromosome from its parent gene. Testis expression is a common initial feature of new gene duplicates (Vinckenbosch et al., 2006), and new gene duplicates appear to frequently acquire regulatory elements that drive testis expression from neighbor genes (Bai et al., 2009). Interestingly, D. melanogaster high throughput expression data show 
that five of the seven genes in the Spd-2B syntenic region (Figure S3A) are most strongly expressed in the testes (Figure S3B-G), consistent with $S p d-2 B$ acquiring enhancers that drive male germline expression from its neighbor genes. Thus, the testis-specific expression pattern of $S p d-2 B$ and the testisbiased expression patterns of several neighbor genes suggests that $S p d-2 B$ picked up testis expression from neighbor gene regulatory elements or from the local chromatin environment at the moment of its birth (Figure 10B). Once Spd-2B had gained spermatocyte expression and lost the C-terminal tail, there would no longer be any evolutionary pressure to maintain the putative autoinhibitory mechanism in male meiosis, thus leading to its loss in Spd-2A (Figure 10B). In summary, our model suggests ancestral (and D. melanogaster) Spd-2 properly organizes PCM in mitosis and meiosis by priming via distinct regulatory sites (Figure $10 \mathrm{C}$ ). In contrast, Spd-2A organizes PCM in mitosis but remains inactive during meiosis due to loss of the relevant regulatory site (Figure 10D). Finally, Spd-2B does not require a priming step due to loss of the C-terminal tail. Therefore, Spd-2B is capable of organizing PCM in both meiosis and mitosis, although under normal conditions it is only expressed in meiotic cells (Figure 10E).

Our discoveries with Spd-2A and Spd-2B suggest that mitotic and male meiotic cell division have subtly different requirements for PCM assembly. One major difference between mitotic and male meiotic cell division in Drosophila is the relatively large size of the meiotic spindle. The large size of the male meiotic spindle is thought to be due in part to high concentrations of tubulin monomers in the spermatocyte cytoplasm required for sperm tail axoneme formation in later spermatogenesis (Lattao et al., 2012). While somatic cells lacking centrosomes can still progress through mitosis using chromatin-nucleated microtubules, male germline cells lacking centrosomes fail to progress through meiosis (Gatti et al., 2012). This failure is thought to reflect a requirement for rapid centrosome-driven polymerization of microtubules in the tubulin-rich spermatocytes. Thus, one possibility is that, in D. melanogaster, Spd-2 activity must be upregulated during meiosis to enhance its ability to nucleate microtubules, whereas, in D. willistoni, Spd-2B is simply specialized for rapid microtubule nucleation to support this distinct requirement of male meiosis.

Our study also revealed several other centrosome gene duplications in the Drosophila lineage. Cnn, which encodes the other major PCM protein in Drosophila, underwent two independent duplications; learning whether these $C n n$ duplicates are specialized in a manner similar to $S p d-2 A$ and $S p d-2 B$ could further support different requirements for PCM in male meiosis. Additionally, of particular interest are duplications of $A s /$ and $C p 110$, which were both duplicated multiple times in the obscura group, providing the potential for multiple specialized forms of these centrosome proteins. Overall, evolution has provided for us a rich resource for uncovering new insights into centrosome biology. 


\section{Methods}

\begin{tabular}{|c|c|c|}
\hline REAGENT or RESOURCE & SOURCE & IDENTIFIER \\
\hline \multicolumn{3}{|l|}{ Antibodies } \\
\hline mouse anti- $\gamma$-Tubulin & Sigma-Aldrich & GTU-88 \\
\hline guinea pig anti-Asl & Rusan Lab & (Klebba et al., 2013) \\
\hline rabbit anti-Cnn & Rusan Lab & (Galletta et al., 2016b) \\
\hline mouse anti-Tubulin & $\begin{array}{l}\text { Developmental Studies } \\
\text { Hybridoma Bank }\end{array}$ & E7 \\
\hline rabbit anti-Phospho-histone $\mathrm{H} 3$ & Millipore & H3S10P \\
\hline $\begin{array}{l}\text { Alexa Fluor } 488 \text { conjugated } \\
\text { secondary antibodies }\end{array}$ & Thermo Fisher Scientific & Variable host and target species \\
\hline $\begin{array}{l}\text { Alexa Fluor } 568 \text { conjugated } \\
\text { secondary antibodies }\end{array}$ & Thermo Fisher Scientific & Variable host and target species \\
\hline $\begin{array}{l}\text { Alexa Fluor } 647 \text { conjugated } \\
\text { secondary antibodies }\end{array}$ & Thermo Fisher Scientific & Variable host and target species \\
\hline \multicolumn{3}{|l|}{ Bacterial and Virus Strains } \\
\hline GC10 Competent Bacteria & Genesee Scientific & Cat \# 42-659 or Cat \# 42-661 \\
\hline \multicolumn{3}{|l|}{$\begin{array}{l}\text { Chemicals, Peptides, and } \\
\text { Recombinant Proteins }\end{array}$} \\
\hline Paraformaldehyde $32 \%$ Solution & Electron Microscopy Sciences & Cat \# 50-980-494 \\
\hline Triton X-100 & Thermo Fisher Scientific & Cat \# BP151-500 \\
\hline Normal Goat Serum & Sigma Aldrich & Cat \# G9023 \\
\hline Aqua-Poly Mount & Polysciences, Inc & Cat \# 18606-20 \\
\hline
\end{tabular}




\begin{tabular}{|c|c|c|}
\hline SF900 II Serum Free Medium & Fisher Scientific & Cat \# 10902104 \\
\hline $\begin{array}{l}\text { Schneider's Drosophila (S2) } \\
\text { Medium }\end{array}$ & Gibco, Thermo Fisher Scientific & Cat \# 21720001 \\
\hline DAPI & Thermo Fisher Scientific & Cat \# D1306 \\
\hline $\begin{array}{l}\text { Phusion High-Fidelity DNA } \\
\text { Polymerase }\end{array}$ & Thermo Fisher Scientific & Cat \# F530L \\
\hline Taq DNA Ligase & New England Biolabs & Cat \# M0208L \\
\hline T5 Exonuclease & Epicentre & Cat \# T5E4111K \\
\hline T4 Polynucleotide Kinase & New England Biolabs & Cat \# M0201S \\
\hline T4 DNA Ligase & New England Biolabs & Cat \# M0202L \\
\hline Critical Commercial Assays & & \\
\hline GeneJet Gel Extraction Kit & Thermo Fisher Scientific & Cat \# FERK0692 \\
\hline GeneJet Plasmid Miniprep Kit & Thermo Fisher Scientific & Cat \# FERK0503 \\
\hline pENTR/D-TOPO Kit & Thermo Fisher Scientific & Cat \# K2400-20 \\
\hline Gateway LR Clonase & Thermo Fisher Scientific & Cat \# 11791043 \\
\hline $\begin{array}{l}\text { Experimental Models: } \\
\text { Organisms/Strains }\end{array}$ & & \\
\hline D. melanogaster: $y w$ & Peifer Lab (UNC-Chapel Hill) & $\mathrm{N} / \mathrm{A}$ \\
\hline D. melanogaster: Spd-2 $2^{23-5711}$ & (Wakimoto et al., 2004) & $\mathrm{N} / \mathrm{A}$ \\
\hline D. melanogaster: $D f(3 L) B S C 561$ & $\begin{array}{l}\text { Bloomington Drosophila Stock } \\
\text { Center }\end{array}$ & BDSC: 25123 \\
\hline D. melanogaster: actin-GAL4 & $\begin{array}{l}\text { Bloomington Drosophila Stock } \\
\text { Center }\end{array}$ & BDSC: 25374 \\
\hline
\end{tabular}




\begin{tabular}{|c|c|c|}
\hline D. melanogaster: topi-GAL4 & $\begin{array}{l}\text { Bloomington Drosophila Stock } \\
\text { Center }\end{array}$ & BDSC: 91776 \\
\hline Recombinant DNA & & \\
\hline pPWG & $\begin{array}{l}\text { Drosophila Genomics Resource } \\
\text { Center }\end{array}$ & DGRC: 1078 \\
\hline pENTR & Thermo Fisher Scientific & Cat \# K2400-20 \\
\hline $\begin{array}{l}\text { pCaSpeR4-attB-Gateway } \\
\text { Cassette }\end{array}$ & Rusan Lab & $\mathrm{N} / \mathrm{A}$ \\
\hline Software and Algorithms & & \\
\hline Nikon Elements & Nikon & \\
\hline FIJI/ImageJ & $\mathrm{NIH}$ & http://fiji.sc/ \\
\hline Excel & Microsoft & $\begin{array}{l}\text { https://products.office.com/en- } \\
\text { us/excel }\end{array}$ \\
\hline Geneious Prime & Biomatters & http://www.geneious.com/ \\
\hline Protein BLAST & NCBI & $\begin{array}{l}\text { https://blast.ncbi.nlm.nih.gov/B } \\
\text { last.cgi/ }\end{array}$ \\
\hline Clustal Omega & EMBL-EBI & $\begin{array}{l}\text { https://www.ebi.ac.uk/Tools/m } \\
\text { sa/clustalo/ }\end{array}$ \\
\hline PAL2NAL & (Suyama et al., 2006) & $\begin{array}{l}\text { http://www.bork.embl.de/pal2 } \\
\underline{\text { nal }}\end{array}$ \\
\hline iTOL & (Letunic and Bork, 2021) & https://itol.embl.de/ \\
\hline Prism 9 & GraphPad & $\begin{array}{l}\text { www.graphpad.com/scientificso } \\
\text { ftware/prism/ }\end{array}$ \\
\hline Photoshop/Illustrator & Adobe & www.adobe.com/uk/products/ \\
\hline
\end{tabular}




\begin{tabular}{|l|l|l|}
\hline & & \\
\hline Other & & \\
\hline Lumox dishes & Sarstedt & Cat \# 94.6077.410 \\
\hline
\end{tabular}

\section{Lead Contact and Materials Availability}

Further information and requests for reagents should be directed to and will be fulfilled by the Lead Contact, N.M.R. (nasser@nih.gov). All unique/stable reagents generated in this study are available upon request without restriction.

\section{BLAST Screen for Gene Duplications}

The protein sequences for 13 major centrosome proteins were obtained from FlyBase (Larkin et al., 2021). For genes with multiple splicing variants, the variant that included all exons was chosen for the BLAST screen (Supplemental File 1). Each protein sequence was then used as a query for Protein BLAST (McGinnis and Madden, 2004) against 35 sequenced Drosophila species (Supplemental File 1). Results were visually inspected, and positive hits were used in a reverse protein BLAST search to determine whether they returned the original query as the top hit. Detailed sequences and records of the screen can be found in Supplemental File 1.

To determine synteny, the gene region including $10 \mathrm{~kb}$ upstream and downstream of either Spd-2A or Spd-2B was obtained from FlyBase and used as query for nucleotide BLAST against the $D$. melanogaster genome.

\section{Sequence Analysis}

The protein sequence and coding sequence for Spd-2 homologs in 7 species were obtained from FlyBase. Protein sequences were first aligned using Clustal Omega with default settings (Sievers et al., 2011). The protein alignment was then converted to a codon alignment using PAL2NAL (Suyama et al., 2006). Gaps were removed from the alignment, and Clustal Omega was used to generate a tree, which was visualized using iTOL (Letunic and Bork, 2021).

To compare both Spd-2A and Spd-2B to D. melanogaster Spd-2, the proteins were divided into an $\mathrm{N}$ terminal half, C-terminal half, and a C-terminal tail that was absent from Spd-2B. Spd-2A and Spd-2B protein segments were aligned with the corresponding segment of Spd-2 using Clustal Omega, and the 
total number of identical amino acids and the total number of inserted or deleted amino acids was recorded.

\section{PCR Screen in Willistoni Group Species}

Drosophila paulistorum, Drosophila equinoxialis, Drosophila tropicalis, Drosophila nebulosa, Drosophila sucinea, and Drosophila virilis strains were obtained from the Drosophila Species Stock Center (Cornell College of Agriculture and Life Science). Genomic DNA was extracted and used to amplify Spd-2A and $S p d-2 B$ from each species. For members of the Willistoni group, primers were based off of $D$. willistoni sequences. Sanger sequencing was used to confirm the identity of PCR products and rule out the possibility of DNA contamination from $D$. willistoni.

\section{D. melanogaster}

Experimental fly crosses were maintained on Bloomington Recipe Fly food from LabExpress and kept at $25^{\circ} \mathrm{C}$. Crosses were either 8 virgin females per vial or 20 virgin females per bottle, with at least half as many males. Controls were $y w$ crossed to $D f(3 L) B S C 561$, and $s p d-2^{-}$were $S p d-2^{23-5711}$ crossed to $D f(3 L) B S C 561$. All new transgenic animals were generated using standard embryo injection protocols by BestGene (Chino Hills, CA).

\section{Generation of Transgenic Drosophila}

For native promoter $S p d-2, S p d-2 A$ and $S p d-2 B$ transgenes, the relevant gene region from the end of the upstream neighbor gene to the beginning of the downstream neighbor gene was PCR amplified from genomic DNA and cloned into pENTR. Gibson assembly was used to insert a GFP-myc or GFP-HA attB cassette in frame at the $3^{\prime}$ end of the coding sequence. Genes were moved to pCaSpeR4 containing an attB recombination site and a Gateway cassette by LR Clonase reactions. pCaSpeR4-attB-Spd-2 constructs were then injected into $D$. melanogaster strain $y^{1} v^{1}$; P[CaryP]attP4O which contains an insulated landing site.

For UAS-Spd-2::GFP, UAS-Spd-2A::GFP, and UAS-Spd-2B::GFP, the relevant coding sequences (including introns) were PCR amplified from genomic DNA and cloned into pENTR using standard Gateway cloning methods. Gateway cloning was used to move constructs from pENTR into pPWG. These constructs were then injected into D. melanogaster strain $y w$.

\section{Immunostaining}


For larval brain experiments, $3^{\text {rd }}$ instar larvae were used. For testes experiments, pupae of various ages were used. Tissues were dissected in SF900 S2 cell media and fixed with 8\% paraformaldehyde in SF900 for 20 minutes at room temperature (RT). Fixed samples were washed three times in PBST (PBS $+0.2 \%$ Triton-X100) and blocked for at least 1 hour in PBST + 5\% normal goat serum (NGS). Blocked samples were incubated in PBST + NGS with primary antibody for either 2 hours at RT or else overnight at $4^{\circ} \mathrm{C}$. Samples were washed five times in PBST + NGS, and then incubated in PBST + NGS with secondary antibody and DAPI for either 2 hours at RT or else overnight at $4^{\circ} \mathrm{C}$. Samples were washed two times in PBST + NGS, two times in PBST, and then several washes in PBS to remove all Triton-X100. Samples were mounted on \#1.5 coverslips and mounted in AquaPoly Mount.

\section{Light Microscopy and Image Analysis}

Imaging was performed using a Nikon W1 spinning disc confocal equipped with a Prime BSI cMOS camera (Photometrics) and a 100X/1.4 NA silicon immersion objective, controlled using Nikon Elements software. Fluorescence intensities were measured and images were prepared for display using ImageJ software (Schneider et al., 2012).

\section{Male Fertility Assay}

Bottles of $y w$ with 10 females and 10 males were flipped daily to prevent over-crowding. Virgin yw females were collected from these bottles and aged for two days. Males of various genotypes to be tested were similarly collected and aged for two days, before being individually placed into vials with two virgin females to mate over the course of four days. After four days, the male was discarded, and females were allowed to lay eggs. At day 17 , the total number of offspring per male was counted.

\section{$\underline{\text { Statistical analysis }}$}

Data analysis was performed using Microsoft Excel and GraphPad Prism. In all graphs, the mean \pm standard deviation is presented. Shapiro-Wilk test was used to test the assumption of normality. ANOVA was used to determine significance, which is reported in Figure legends. 


\section{Acknowledgements}

We thank Bloomington Drosophila Stock Center and the National Drosophila Species Stock Center at Cornell College of Agricultural Sciences for fly stocks, and Developmental Studies Hybridoma Bank for antibodies. Carey Fagerstrom performed all cloning. We thank Alex Kelly, Takashi Akera, Matthew Hannaford, Ramya Varadarajan, and Chaitali Khan for helpful discussion. 


\section{References}

Alvarez-Rodrigo, I., Steinacker, T.L., Saurya, S., Conduit, P.T., Baumbach, J., Novak, Z.A., Aydogan, M.G., Wainman, A., and Raff, J.W. (2019). Evidence that a positive feedback loop drives centrosome maturation in fly embryos. Elife 8. 10.7554/eLife.50130.

Bai, Y., Casola, C., and Betrán, E. (2009). Quality of regulatory elements in Drosophila retrogenes. Genomics 93, 83-89. 10.1016/j.ygeno.2008.09.006.

Chen, J.V., Buchwalter, R.A., Kao, L.R., and Megraw, T.L. (2017). A Splice Variant of Centrosomin Converts Mitochondria to Microtubule-Organizing Centers. Curr Biol 27, 1928-1940.e1926.

10.1016/j.cub.2017.05.090.

Conduit, P.T., Brunk, K., Dobbelaere, J., Dix, C.I., Lucas, E.P., and Raff, J.W. (2010). Centrioles regulate centrosome size by controlling the rate of Cnn incorporation into the PCM. Curr Biol 20, 2178-2186. 10.1016/j.cub.2010.11.011.

Conduit, P.T., Richens, J.H., Wainman, A., Holder, J., Vicente, C.C., Pratt, M.B., Dix, C.I., Novak, Z.A., Dobbie, I.M., Schermelleh, L., and Raff, J.W. (2014). A molecular mechanism of mitotic centrosome assembly in Drosophila. Elife 3, e03399. 10.7554/eLife.03399.

Conduit, P.T., Wainman, A., and Raff, J.W. (2015). Centrosome function and assembly in animal cells. Nat Rev Mol Cell Biol 16, 611-624. 10.1038/nrm4062.

Decker, M., Jaensch, S., Pozniakovsky, A., Zinke, A., O'Connell, K.F., Zachariae, W., Myers, E., and Hyman, A.A. (2011). Limiting amounts of centrosome material set centrosome size in C. elegans embryos. Curr Biol 21, 1259-1267. 10.1016/j.cub.2011.06.002.

Des Marais, D.L., and Rausher, M.D. (2008). Escape from adaptive conflict after duplication in an anthocyanin pathway gene. Nature 454, 762-765. 10.1038/nature07092. 
Dix, C.I., and Raff, J.W. (2007). Drosophila Spd-2 Recruits PCM to the Sperm Centriole, but Is Dispensable for Centriole Duplication. Current Biology 17, 1759-1764. https://doi.org/10.1016/j.cub.2007.08.065.

Ezawa, K., Ikeo, K., Gojobori, T., and Saitou, N. (2011). Evolutionary patterns of recently emerged animal duplogs. Genome Biol Evol 3, 1119-1135. 10.1093/gbe/evr074.

Galletta, B.J., Fagerstrom, C.J., Schoborg, T.A., McLamarrah, T.A., Ryniawec, J.M., Buster, D.W., Slep, K.C., Rogers, G.C., and Rusan, N.M. (2016a). A centrosome interactome provides insight into organelle assembly and reveals a non-duplication role for Plk4. Nat Commun 7, 12476. 10.1038/ncomms12476.

Galletta, B.J., Guillen, R.X., Fagerstrom, C.J., Brownlee, C.W., Lerit, D.A., Megraw, T.L., Rogers, G.C., and Rusan, N.M. (2014). Drosophila pericentrin requires interaction with calmodulin for its function at centrosomes and neuronal basal bodies but not at sperm basal bodies. Molecular Biology of the Cell 25, 2682-2694. 10.1091/mbc.e13-10-0617.

Galletta, B.J., Jacobs, K.C., Fagerstrom, C.J., and Rusan, N.M. (2016b). Asterless is required for centriole length control and sperm development. J Cell Biol 213, 435-450. 10.1083/jcb.201501120.

Gatti, M., Bucciarelli, E., Lattao, R., Pellacani, C., Mottier-Pavie, V., Giansanti, M.G., Somma, M.P., and Bonaccorsi, S. (2012). The relative roles of centrosomal and kinetochore-driven microtubules in Drosophila spindle formation. Exp Cell Res 318, 1375-1380. 10.1016/j.yexcr.2012.05.001.

Giansanti, M.G., Bucciarelli, E., Bonaccorsi, S., and Gatti, M. (2008). Drosophila SPD-2 Is an Essential Centriole Component Required for PCM Recruitment and Astral-Microtubule Nucleation. Current Biology 18, 303-309. https://doi.org/10.1016/j.cub.2008.01.058.

Gomez-Ferreria, M.A., and Sharp, D.J. (2008). Cep192 and the generation of the mitotic spindle. Cell Cycle 7, 1507-1510. 10.4161/cc.7.11.5957.

Goshima, G., Wollman, R., Goodwin, S.S., Zhang, N., Scholey, J.M., Vale, R.D., and Stuurman, N. (2007). Genes required for mitotic spindle assembly in Drosophila S2 cells. Science 316, 417-421. $10.1126 /$ science.1141314. 
Greenan, G., Brangwynne, C.P., Jaensch, S., Gharakhani, J., Jülicher, F., and Hyman, A.A. (2010).

Centrosome size sets mitotic spindle length in Caenorhabditis elegans embryos. Curr Biol 20, 353-358.

10.1016/j.cub.2009.12.050.

Hughes, A.L. (1994). The evolution of functionally novel proteins after gene duplication. Proc Biol Sci 256, 119-124. 10.1098/rspb.1994.0058.

Kemp, C.A., Kopish, K.R., Zipperlen, P., Ahringer, J., and O'Connell, K.F. (2004). Centrosome maturation and duplication in C. elegans require the coiled-coil protein SPD-2. Dev Cell 6, 511-523. 10.1016/s15345807(04)00066-8.

Klebba, J.E., Buster, D.W., Nguyen, A.L., Swatkoski, S., Gucek, M., Rusan, N.M., and Rogers, G.C. (2013). Polo-like kinase 4 autodestructs by generating its Slimb-binding phosphodegron. Curr Biol 23, 22552261. 10.1016/j.cub.2013.09.019.

Kwon, M., and Scholey, J.M. (2004). Spindle mechanics and dynamics during mitosis in Drosophila. Trends Cell Biol 14, 194-205. 10.1016/j.tcb.2004.03.003.

Larkin, A., Marygold, S.J., Antonazzo, G., Attrill, H., Dos Santos, G., Garapati, P.V., Goodman, J.L., Gramates, L.S., Millburn, G., Strelets, V.B., et al. (2021). FlyBase: updates to the Drosophila melanogaster knowledge base. Nucleic Acids Res 49, D899-d907. 10.1093/nar/gkaa1026.

Lattao, R., Bonaccorsi, S., and Gatti, M. (2012). Giant meiotic spindles in males from Drosophila species with giant sperm tails. J Cell Sci 125, 584-588. 10.1242/jcs.101469.

Lerit, D.A., and Poulton, J.S. (2016). Centrosomes are multifunctional regulators of genome stability. Chromosome Res 24, 5-17. 10.1007/s10577-015-9506-4.

Letunic, I., and Bork, P. (2021). Interactive Tree Of Life (iTOL) v5: an online tool for phylogenetic tree display and annotation. Nucleic Acids Res 49, W293-w296. 10.1093/nar/gkab301. 
McGinnis, S., and Madden, T.L. (2004). BLAST: at the core of a powerful and diverse set of sequence analysis tools. Nucleic Acids Res 32, W20-25. 10.1093/nar/gkh435.

Meng, L., Park, J.-E., Kim, T.-S., Lee, E.H., Park, S.-Y., Zhou, M., Bang, J.K., and Lee, K.S. (2015). Bimodal Interaction of Mammalian Polo-Like Kinase 1 and a Centrosomal Scaffold, Cep192, in the Regulation of Bipolar Spindle Formation. Molecular and Cellular Biology 35, 2626-2640. doi:10.1128/MCB.00068-15.

Modarressi, M.H., Cameron, J., Taylor, K.E., and Wolfe, J. (2001). Identification and characterisation of a novel gene, TSGA10, expressed in testis. Gene 262, 249-255. 10.1016/s0378-1119(00)00519-9.

Ohkura, H. (2015). Meiosis: an overview of key differences from mitosis. Cold Spring Harb Perspect Biol 7. 10.1101/cshperspect.a015859.

Ohno, S. (1970). Evolution by gene duplication (Allen \& Unwin;Springer-Verlag).

Pelletier, L., Ozlü, N., Hannak, E., Cowan, C., Habermann, B., Ruer, M., Müller-Reichert, T., and Hyman, A.A. (2004). The Caenorhabditis elegans centrosomal protein SPD-2 is required for both pericentriolar material recruitment and centriole duplication. Curr Biol 14, 863-873. 10.1016/j.cub.2004.04.012.

Rieckhoff, E.M., Ishihara, K., and Brugués, J. (2019). How to tune spindle size relative to cell size? Curr Opin Cell Biol 60, 139-144. 10.1016/j.ceb.2019.06.007.

Rogers, R.L., and Hartl, D.L. (2012). Chimeric genes as a source of rapid evolution in Drosophila melanogaster. Mol Biol Evol 29, 517-529. 10.1093/molbev/msr184.

Russo, C.A.M., Mello, B., Frazão, A., and Voloch, C.M. (2013). Phylogenetic analysis and a time tree for a large drosophilid data set (Diptera: Drosophilidae). Zoological Journal of the Linnean Society 169, 765775. 10.1111/zoj12062.

Schneider, C.A., Rasband, W.S., and Eliceiri, K.W. (2012). NIH Image to ImageJ: 25 years of image analysis. Nat Methods 9, 671-675. 10.1038/nmeth.2089. 
Sha, Y.W., Sha, Y.K., Ji, Z.Y., Mei, L.B., Ding, L., Zhang, Q., Qiu, P.P., Lin, S.B., Wang, X., Li, P., et al. (2018). TSGA10 is a novel candidate gene associated with acephalic spermatozoa. Clin Genet 93, 776-783.

10.1111/cge.13140.

Sievers, F., Wilm, A., Dineen, D., Gibson, T.J., Karplus, K., Li, W., Lopez, R., McWilliam, H., Remmert, M., Söding, J., et al. (2011). Fast, scalable generation of high-quality protein multiple sequence alignments using Clustal Omega. Mol Syst Biol 7, 539. 10.1038/msb.2011.75.

Suyama, M., Torrents, D., and Bork, P. (2006). PAL2NAL: robust conversion of protein sequence alignments into the corresponding codon alignments. Nucleic Acids Res 34, W609-612.

10.1093/nar/gkl315.

Taylor, J.S., and Raes, J. (2004). Duplication and divergence: the evolution of new genes and old ideas. Annu Rev Genet 38, 615-643. 10.1146/annurev.genet.38.072902.092831.

Vasquez-Limeta, A., and Loncarek, J. (2021). Human centrosome organization and function in interphase and mitosis. Semin Cell Dev Biol 117, 30-41. 10.1016/j.semcdb.2021.03.020.

Vinckenbosch, N., Dupanloup, I., and Kaessmann, H. (2006). Evolutionary fate of retroposed gene copies in the human genome. Proc Natl Acad Sci U S A 103, 3220-3225. 10.1073/pnas.0511307103.

Wakimoto, B.T., Lindsley, D.L., and Herrera, C. (2004). Toward a comprehensive genetic analysis of male fertility in Drosophila melanogaster. Genetics 167, 207-216. 10.1534/genetics.167.1.207. 
Centriole Bridge

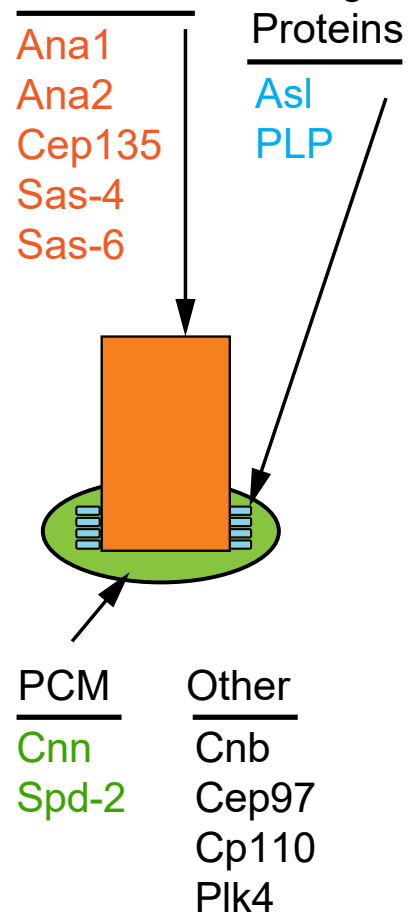

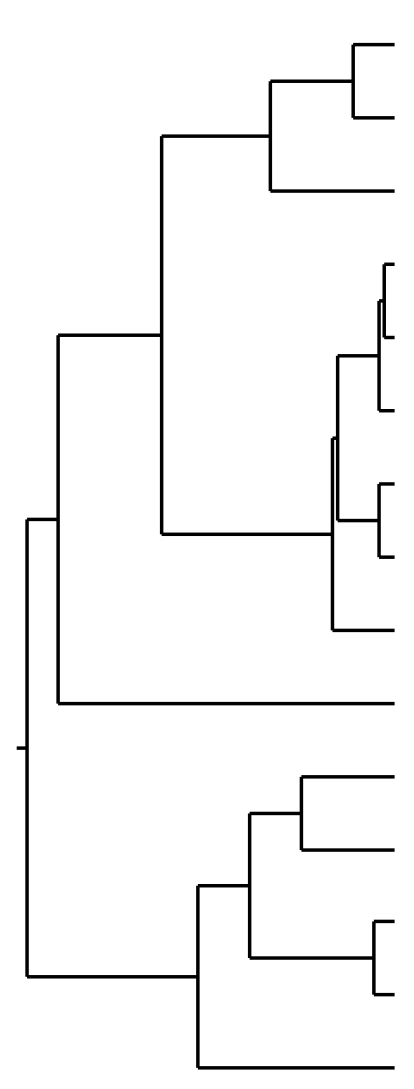

D. melanogaster

D. yakuba

D. rhopaloa

D. pseudoobscura

D. persimilis

D. miranda

D. guanche

D. subobscura

D. obscura

D. willistoni

D. mojavensis

D. hydei

D. virilis

D. novamexicana

D. innubila

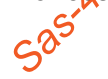

$\partial^{\mathrm{S}}$

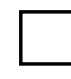

$\mathrm{A} \mid \mathrm{B}$

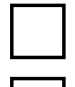

$\square$

$\square$

$\square$ B1|B2|B3|C

$\square$\begin{tabular}{|l|l|l|l|l|}
\hline$A$ & $C_{1}$ & $C_{2}$ & $C_{3}$ \\
\hline
\end{tabular}

\begin{tabular}{|l|l|}
\hline $\mathrm{A}$ & $\mathrm{C}$ \\
\hline
\end{tabular}

\begin{tabular}{|l|l|}
\hline $\mathrm{A}$ & $\mathrm{C}$ \\
\hline
\end{tabular}

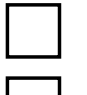

$\square$

$\square$

\begin{tabular}{|l|l|l|}
\hline $\mathrm{A}$ & $\mathrm{B}$ & $\mathrm{C}$ \\
\hline
\end{tabular}

\begin{tabular}{|l|l|}
\hline$A$ & $B$ \\
\hline
\end{tabular}

\begin{tabular}{|l|l|}
\hline$A$ & $B$ \\
\hline
\end{tabular}

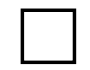

$\square$
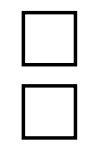

\begin{tabular}{|l|l|}
\hline$A$ & $B$ \\
\hline
\end{tabular}

\begin{tabular}{|l|l|}
\hline $\mathrm{A}$ & $\mathrm{C}$ \\
\hline
\end{tabular}

\begin{tabular}{|l|l|}
\hline$A$ & $C$ \\
\hline
\end{tabular}
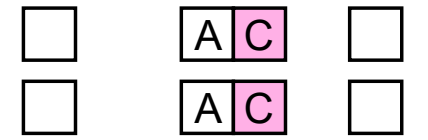

\begin{tabular}{|l|l|}
\hline$A$ & $C$ \\
\hline
\end{tabular}

$\square$

$\square$

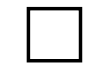

$\square$

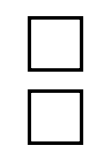

\begin{tabular}{|l|l|l|}
\hline $\mathrm{A}$ & $\mathrm{C}$ \\
\hline
\end{tabular}

$\mathrm{A} \mid \mathrm{C}$

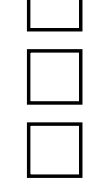

\begin{tabular}{|l|l|l|}
\hline$A$ & $D$ & $E$ \\
\hline
\end{tabular}

$\mathrm{A} \mid \mathrm{D}$

$\mathrm{A} \mid \mathrm{D}$

$\mathrm{A} \mid \mathrm{D}$
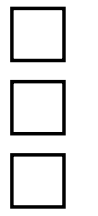

$\square$

$\square$

$\square$

Figure 1 - Centrosome Gene Duplications in the Drosophila lineage 


\section{Figure 1 - Centrosome gene duplications in the Drosophila lineage}

(A) Diagram of a centrosome showing proteins used in a BLAST screen for centrosome gene duplications. Categories included core centriole proteins (orange), bridge proteins (blue), and PCM proteins (green).

(B) Summary of BLAST screen results. Only genes and species where duplications were found are shown. White boxes represent single copy genes. Lettered boxes represent duplicated genes: a white box with " $A$ " represents the orthologs of the single copy gene, while each colored boxes with a letter represents a unique duplication. For Spd-2, duplication " $\mathrm{X}$ " in D. melanogaster represents a chimeric pseudogene. For asl, duplications " $\mathrm{B}$ " and " $\mathrm{C}$ " were subsequently re-duplicated multiple times, and these duplications are represented by boxes with a letter and number. 

(which was not certified by peer review) is the author/funder. All rights reserved. No reuse allowed without permission.

\section{Chr. 3L \\ Chr. 2R}

\section{Spd-2}

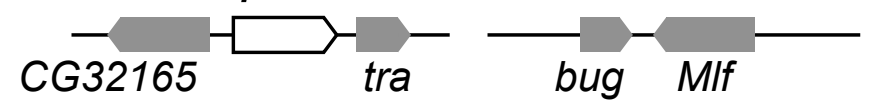

\section{D. willistoni}

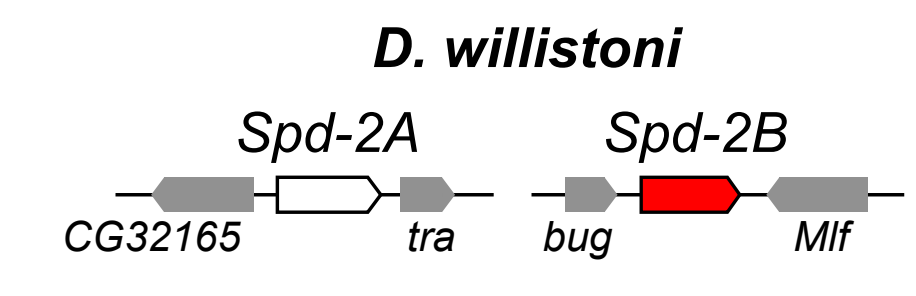

B

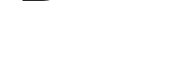

C
D
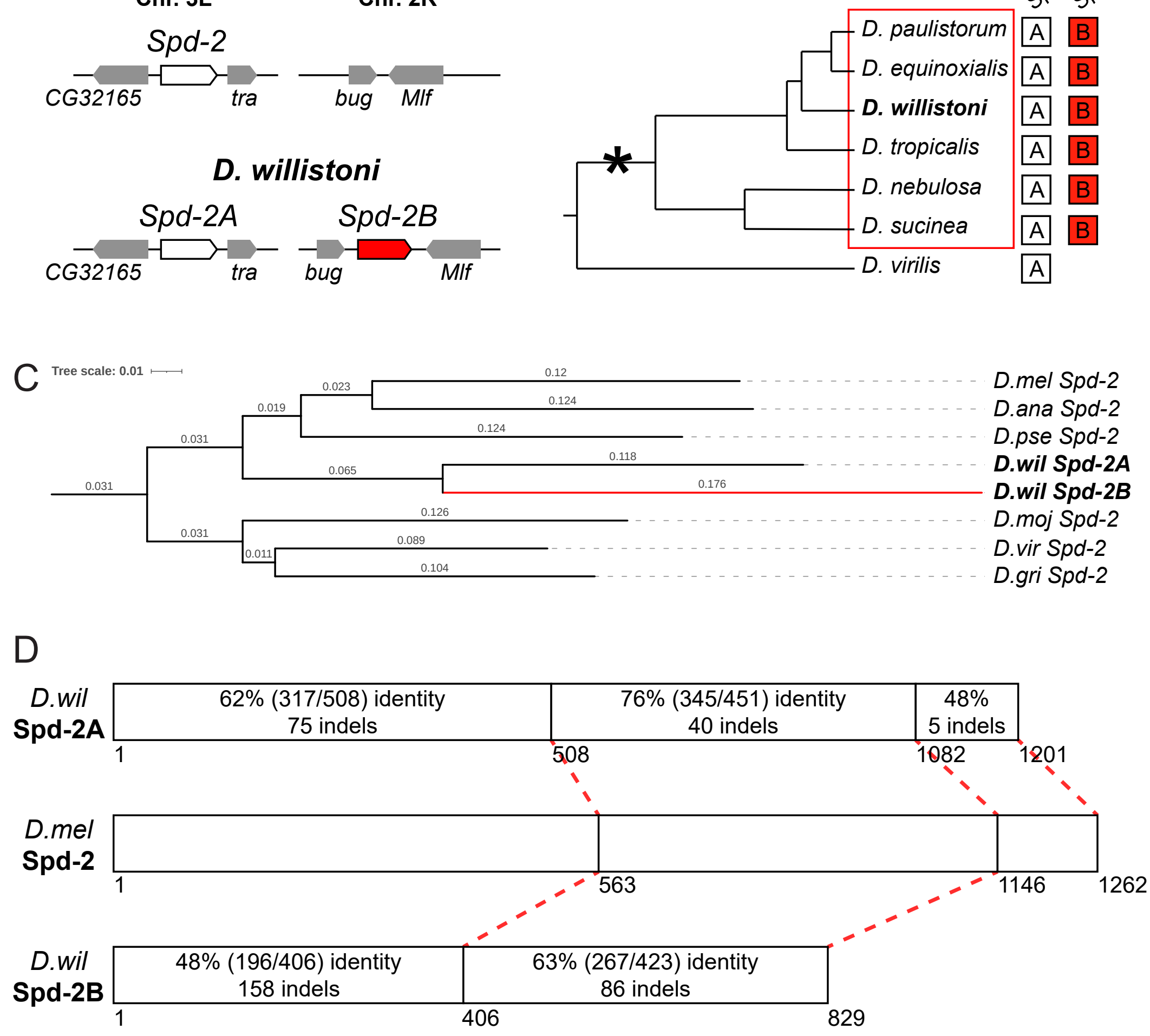

Figure 2 - Drosophila willistoni Spd-2B is a Conserved, Rapidly Evolving Duplication of Spd-2 


\section{Figure 2-Drosophila willistoni Spd-2B is a conserved, rapidly evolving duplication of Spd-2}

(A) Top: D. melanogaster gene regions of Spd-2 locus (Chromosome $3 \mathrm{~L}$, left) and the syntenic locus to where Spd-2B is inserted in $D$. willistoni (Chromosome 2R, right). Bottom: $D$. willistoni gene regions of $S p d-2 A$ (left) and $S p d-2 B$ (right). Adapted from Flybase.

(B) Willistoni group lineage showing presence of both $S p d-2 A$ and $S p d-2 B$ in multiple species confirmed by PCR test. Note the absence of $S p d-2 B$ in the outgroup species Drosophila virilis.

(C) Phylogenetic tree based on a trimmed codon alignment of Spd-2, Spd-2A and Spd-2B. The branch length of $S p d-2 B$ (red branch) is significantly longer than $S p d-2 A$, indicating rapid divergence.

(D) Diagrammatic representation of $D$. melanogaster Spd-2 (middle) compared to $D$. willistoni Spd-2A (top) and Spd-2B (bottom) proteins. A comparison of amino acid identity and indels to $D$. melanogaster Spd-2 are shown for both Spd-2A and Spd-2B. Spd-2B is completely lacking the Spd-2 C-terminal tail. 

(which was not certified by peer review) is the author/funder. All rights resecohttioofuse allowed without permissigg pd-2

A

mel(p)-Spd-2::GFP; spd-2

B

wil(p)-Spd-2A::GFP; spd-2

C

wil(p)-Spd-2B::GFP; $s p d-2$
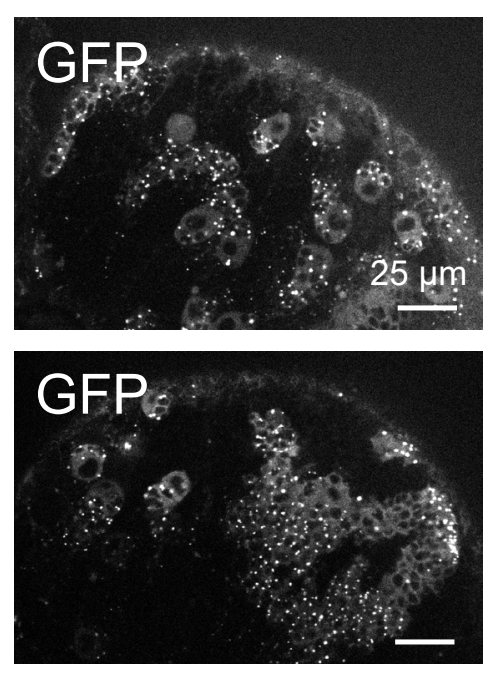

\section{GFP}

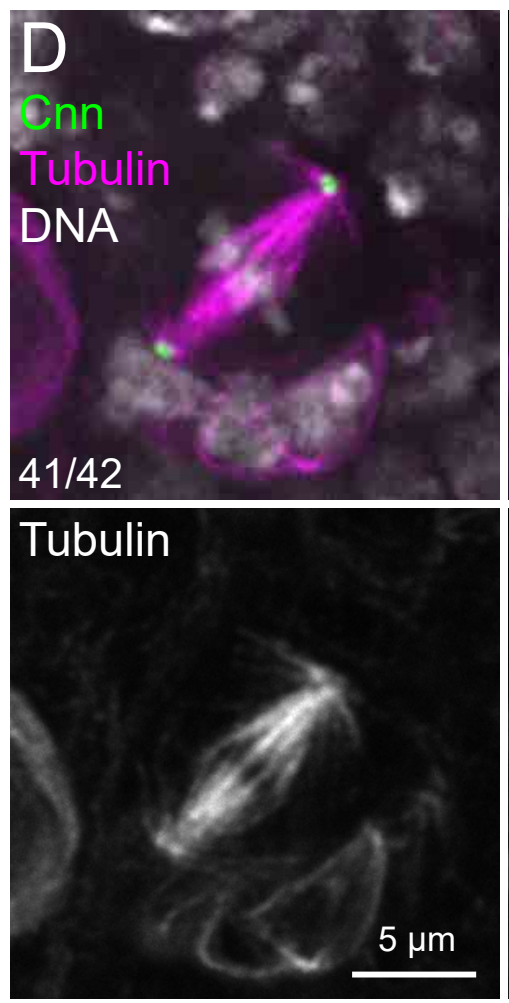

wil(p)-Spd-2B::GFP; spd-2

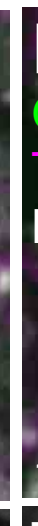

$\mathrm{H}$

GFP

Tubufing

DNA

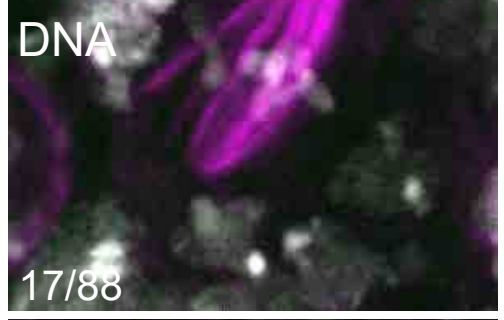

Tubulin

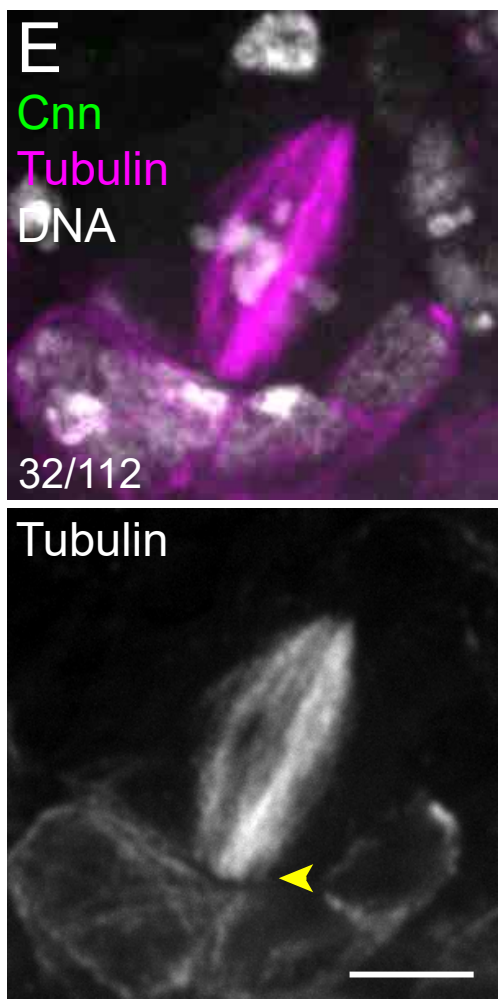

actin-GAL4 > Spd-2B::GFP; spd-2-

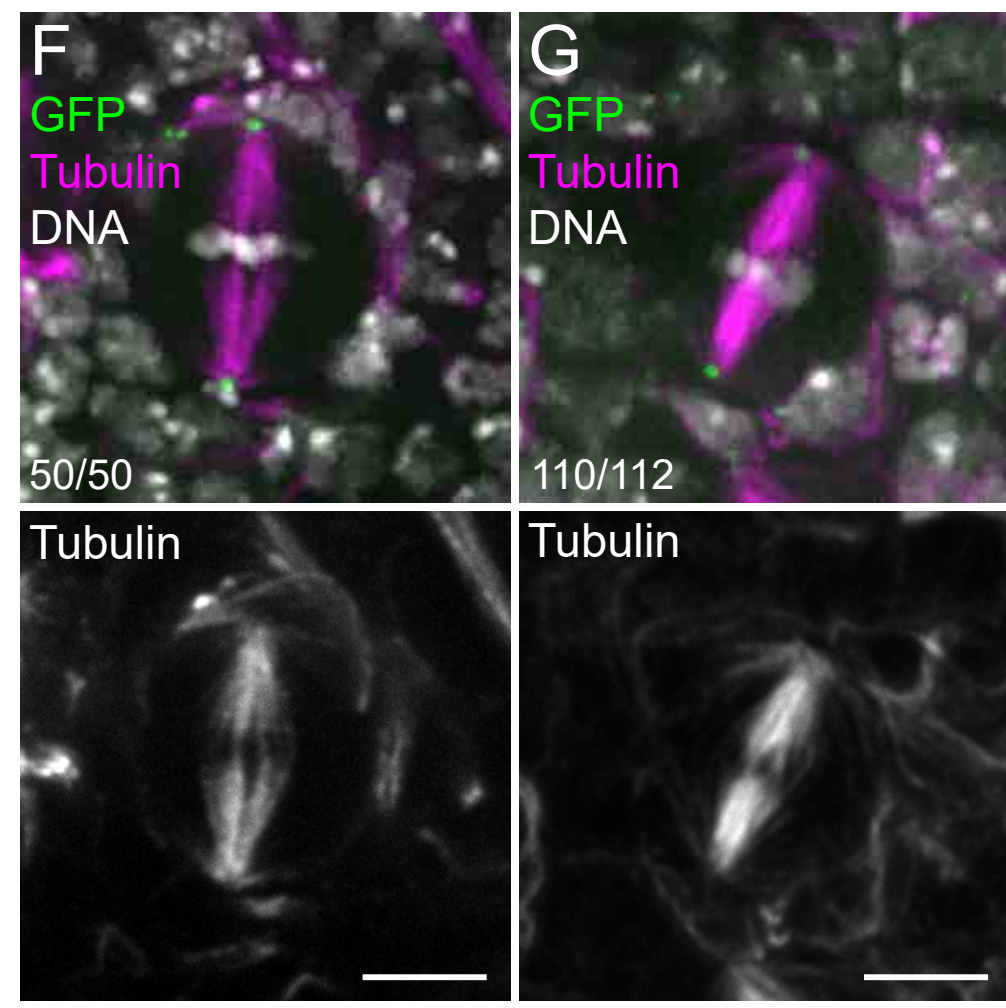

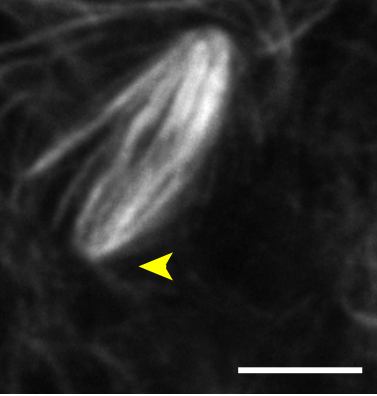

Figure 3 - Spd-2A and Spd-2B Can Organize the Mitotic Spindle in Larval Brain Neuroblasts 


\section{Figure 3-Spd-2A and Spd-2B can organize the mitotic spindle in larval brain neuroblasts}

(A-C) Micrographs of unfixed $3^{\text {rd }}$ instar larval brains expressing $D$. melanogaster Spd-2::GFP (A) and D. willistoni Spd-2A::GFP (B) or Spd-2B::GFP (C).

(D-E) Micrographs of neuroblast metaphase spindles of controls (D) and spd-2 mutants (E), stained for Cnn (green), Tubulin (magenta), and DNA (white). Single channel micrographs of Tubulin alone are shown below. Controls show normal morphology with a tight spindle that is properly focused at the poles (1/42 unfocused). spd-2 mutants have a barrel shaped spindle with frequently unfocused poles (32/112 unfocused; yellow arrowhead).

(F-I) Micrographs of neuroblast metaphase spindles from spd-2 mutants with rescue constructs mel(p)Spd-2::GFP (F), wil(p)-Spd-2A::GFP (G), wil(p)-Spd-2B::GFP (H) , and actin-GAL4 > UAS-Spd-2B::GFP (I) stained for Tubulin (magenta), and DNA (white), and expressing Spd-2(A/B)::GFP (green). Both Spd$2:: G F P$ and Spd-2A::GFP are expressed in neuroblasts and rescue spd-2 ${ }^{-}$spindle morphology (0/50 and 2/112 unfocused, respectively; F, G). Spd-2B::GFP is not detectable in neuroblasts, and fails to rescue spd-2- spindle morphology (17/88 unfocused; $H$, yellow arrowhead). When expressed via actin-GAL4, Spd-2B::GFP localizes to the poles and properly organizes the spindle (2/50 unfocused; I). 


\section{Figure 4 - Spd-2A and Spd-2B can recruit centrosomal $p$-Tubulin and Cnn in larval brain} neuroblasts

(A-B) Micrographs of metaphase neuroblasts from controls (A) and spd-2- mutants mutants (B), stained for Asl (red), DNA (white), and either $\psi$-Tubulin (blue, left panels) or Cnn (blue, right panels). A zoom to the apical centrosome with isolated Asl and $y$-Tubulin or Cnn channels are shown to the right.

(C-F) Micrographs of metaphase neuroblasts from spd-2 mutants mutants with rescue constructs mel(p)-Spd-2::GFP (C), wil(p)-Spd-2A::GFP (D), wil(p)-Spd-2B::GFP (E), or actin-GAL4 > UAS-Spd-2B::GFP (F) expressing Spd-2(A/B)::GFP (green) and stained Asl (red), DNA (white), and either $\gamma$-Tubulin (blue, left panels) or Cnn (blue, right panels).

(G-I) Quantification of the number of Asl dots (G), Y-Tubulin dots (H), and Cnn dots (I) per neuroblast for genotypes in A-F. Error bars represent standard deviation. Significance tested via ordinary one-way ANOVA. ns $=$ not significant; $* * * * p<0.001 ; * * p<0.01$. 

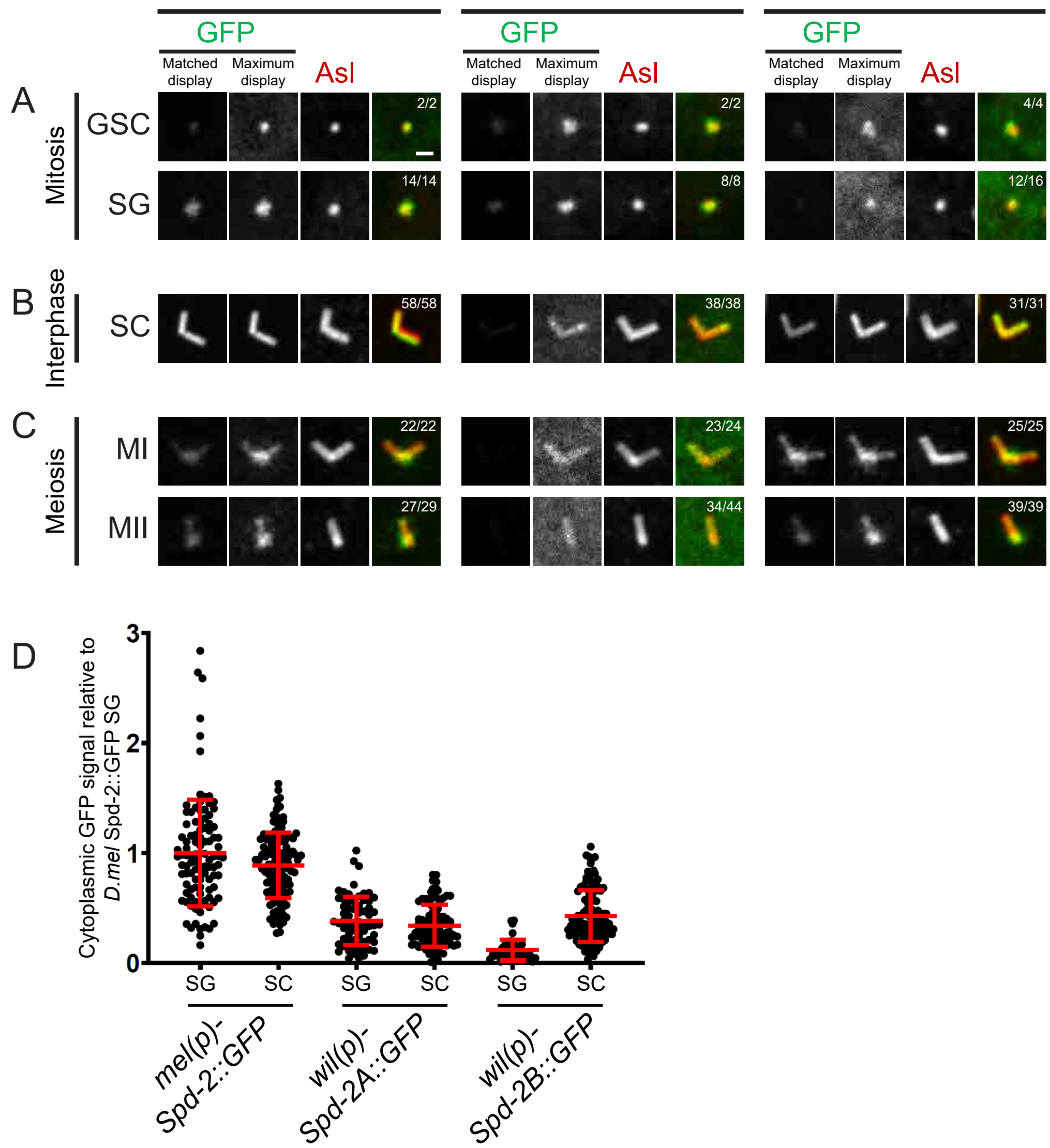

Figure 5 - Spd-2A and Spd-2B Have Complementary Centrosomal Localizations in Spermatogenesis 


\section{Figure 5 - Spd-2A and Spd-2B have complementary centriolar localizations in spermatogenesis}

$(\mathrm{A}, \mathrm{B}, \mathrm{C})$ Micrographs of $s p d-2^{-}$mutants with mel(p)-Spd-2::GFP, wil(p)-Spd-2A::GFP, or wil(p)-Spd2B::GFP (GFP in green) stained for Asl (red) in various stages of spermatogenesis: (A) mitotic stages of male germline stem cells (GSC) and spermatogonia (SG); (B) interphase stage of mature spermatocytes (SC); and (C) spermatocytes in meiosis I (MI); meiosis II (MII). GFP channels are displayed in two ways: first, using the same display range for all images (Matched display, left), and, second, such that the dynamic range of each image corresponds to the brightest and dimmest pixels (Maximum display, right). Note that Spd-2::GFP has robust centrosomal localization throughout spermatogenesis (A). In constrast, Spd-2A::GFP localizes to the centrosome early (B), whereas Spd-2B::GFP localizes to the centrosome late in spermatogenesis (C).

(D) Quantification of cytoplasmic Spd-2(A/B)::GFP in spermatogonia (SG) and spermatocytes (SC). All values are normalized to $D$. melanogaster Spd-2 in SG. Note that Spd-2A cytoplasmic levels remain relatively consistent throughout spermatogenesis, while centrosomal levels appear reduced during meiosis. 


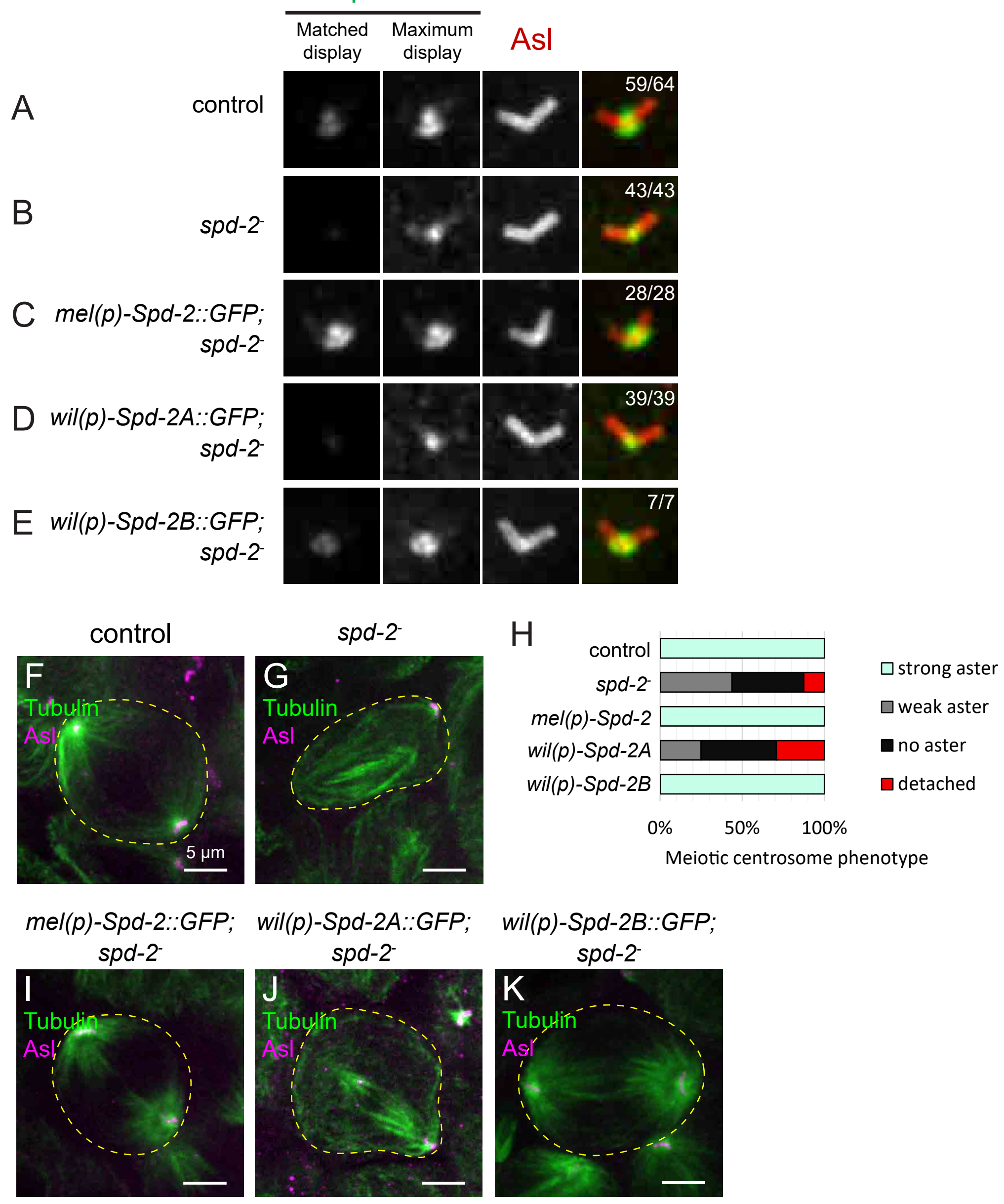

Figure 6 - Spd-2B, But Not Spd-2A, Recruits $\gamma$-Tubulin and Organizes Spindles During Meiosis 


\section{Figure 6 -Spd-2B, but not Spd-2A, recruits $\gamma$-Tubulin and organizes spindles during meiosis}

(A-E) Micrographs of control (A), spd-2- mutant (B), and mel(p)-Spd-2::GFP; spd-2- (C) wil(p)-Spd-2A::GFP; spd-2- (D) and wil(p)-Spd-2B::GFP; spd-2- (E) spermatocyte centrosomes during meiosis I, stained for $\mathrm{Y}^{-}$ Tubulin (green) and Asl (red). $\nu$-tubulin channels are displayed in two ways: first, using the same display range for all images (Matched display, left), and, second, such that the dynamic range of each image corresponds to the brightest and dimmest pixels (Maximum display, right). Note that controls $(A)$ and Spd-2B::GFP rescues (E) recruit similar levels of $\gamma$-Tubulin to the centrosomes, whereas both Spd-2 mutants (B) and Spd-2A::GFP (D) rescues fail to properly recruit $\gamma$-Tubulin.

$(F, G)$ Micrographs of control (F) and spd-2- mutant (G) spermatocytes during meiosis I, stained for Tubulin (green) and Asl (magenta).

(H) Quantification of centrosome phenotypes. Strong aster corresponds to centrosomes with full asters as seen in (F); weak asters correspond to centrosomes with only a few microtubules as seen in (G); no asters corresponds to centrosomes with no visible microtubules; and detached corresponds to centrosomes that have detached from the poles.

(I-K) Micrographs of spd-2- mutants with native promoter rescues mel(p)-Spd-2::GFP (I), wil(p)-Spd2A::GFP (J), or wil(p)-Spd-2B::GFP (K) stained for Tubulin (green) and Asl (magenta). 
A

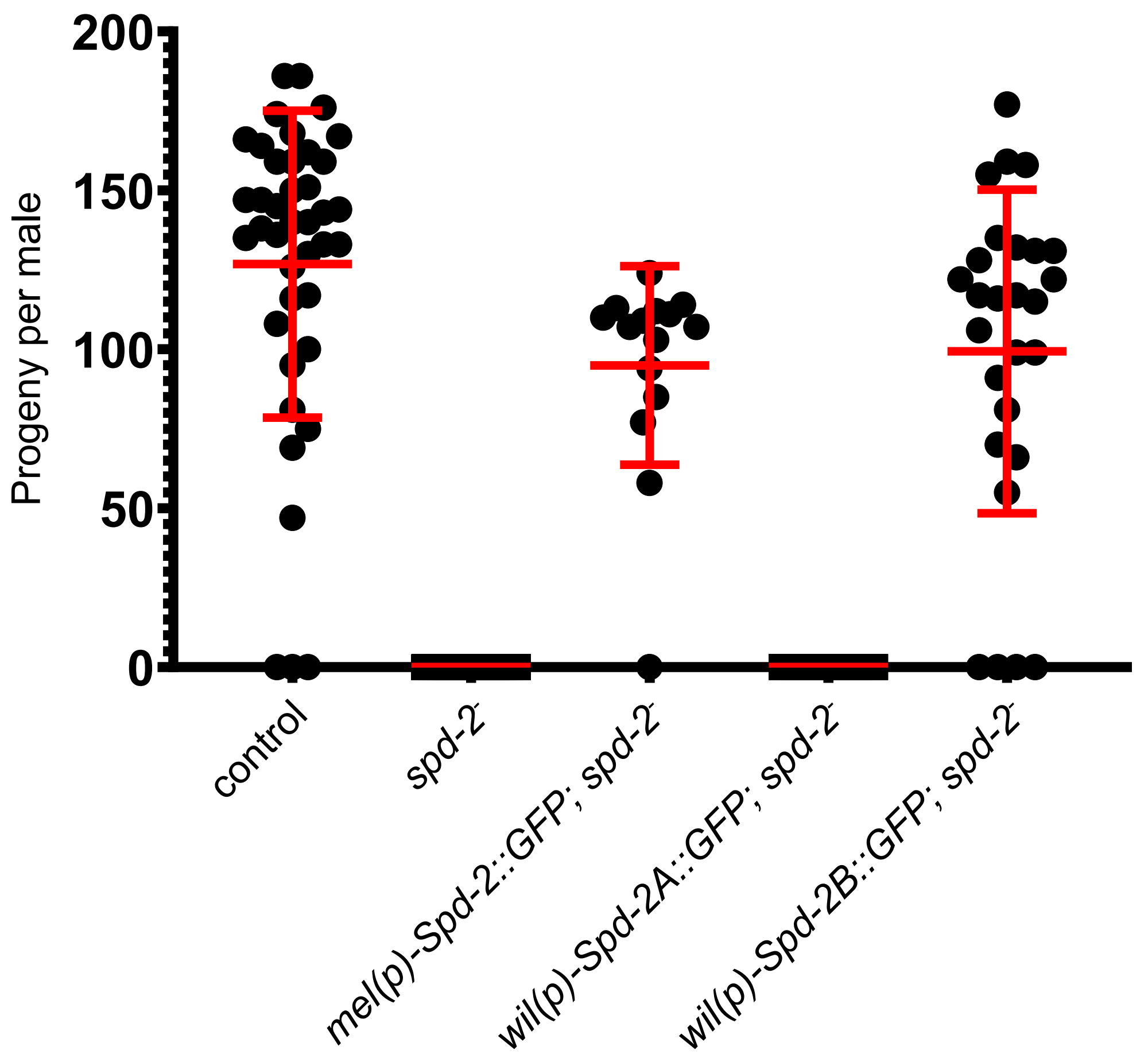

Figure $7-S p d-2 B$ is Required for Male Fertility but $S p d-2 A$ is Dispensable 
bioRxiv preprint doi: https://doi.org/10.1101/2021.10.27.466145; this version posted October 28, 2021. The copyright holder for this preprint (which was not certified by peer review) is the author/funder. All rights reserved. No reuse allowed without permission.

Figure $7-S p d-2 B$ is required for male fertility but $S p d-2 A$ is dispensable

(A) Male fertility of control, spd-2 mutant, and native promoter rescues. Single males were mated to two virgin females, and the total number of offspring per male was counted. Controls, mel(p)-Spd-2::GFP rescues and wil(p)-Spd-2B::GFP rescues were fertile, whereas spd-2 mutants and wil(p)-Spd-2A::GFP rescues were completely sterile. 
bioRxiv preprint doi: https://doi.org/10.1101/2021.10.27.466145; this version posted October 28, 2021. The copyright holder for this preprint

A topi-GAL4 > B B topi-GAL4> C C topi-GAL4 > Spd-2::GFP; spd-2

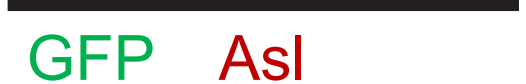

Maximum display
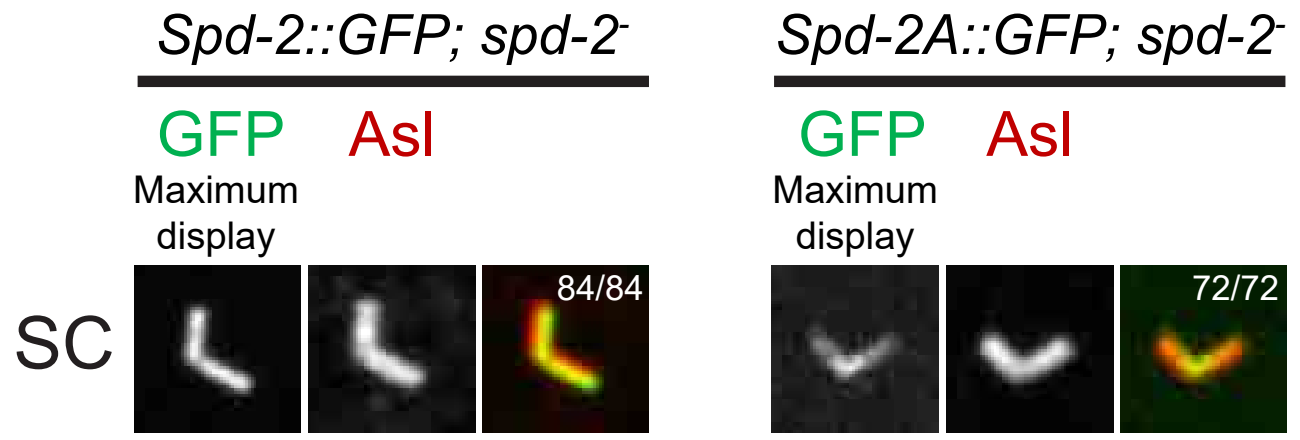
Spd-2B::GFP; spd-2-

Maximum display
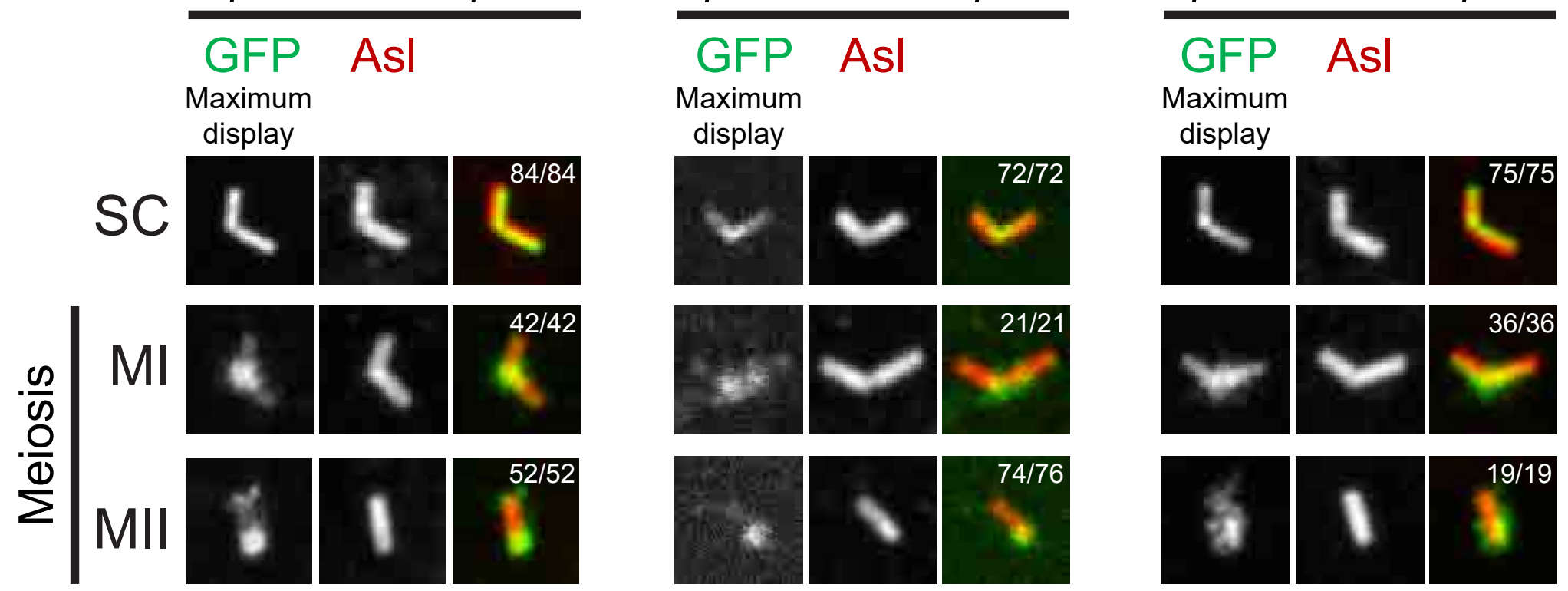

$36 / 36$
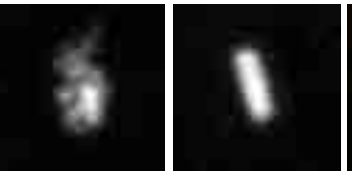

$19 / 19$

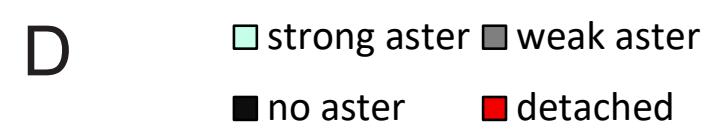

topi-GAL4 > topi-GAL4 > topi-GAL4 > Spd-2::GFP; spd-2- Spd-2A::GFP; spd-2- Spd-2B::GFP; spd-2-
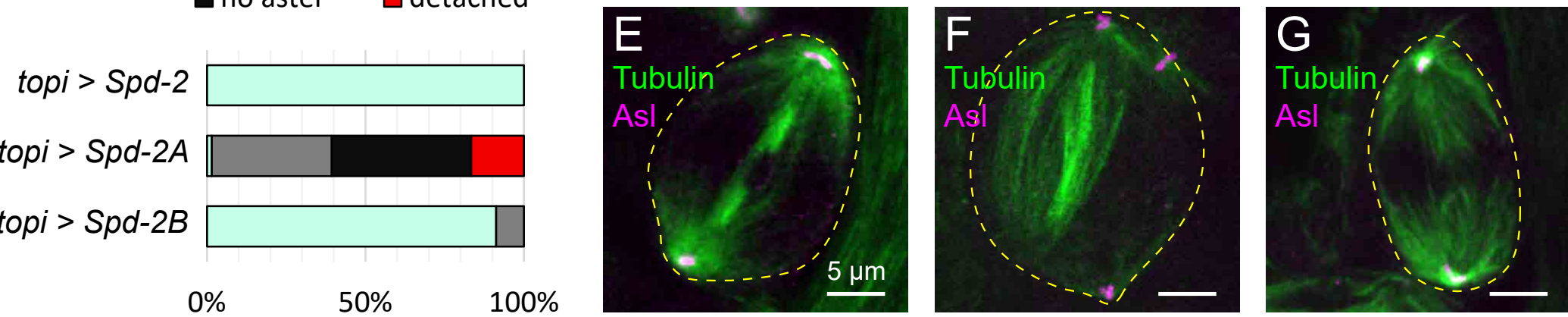

Meiotic centrosome phenotype

\section{Figure 8 - Spd-2A Fails to Properly Localize to Meiotic Centrosomes}




\section{Figure 8 - Spd-2A fails to properly localize to meiotic centrosomes}

(A-C) Micrographs of topi-GAL4 driving UAS-Spd-2::GFP (A), UAS-Spd-2A::GFP (B) and UAS-Spd-2B::GFP (C) showing Spd-2(A/B)::GFP localization to centrosomes in spermatocytes. GFP channels are displayed such that the dynamic range corresponds to the brightest and dimmest pixels. Note that both Spd-2 (A) and Spd-2B (C) are robustly recruited to spermatocyte centriole, whereas Spd-2A (B) is barely detectable. SC = spermatocytes; MI = meiosis I; MII = meiosis II.

(D) Quantification of centrosome phenotypes for topi-GAL4 rescues. Strong aster corresponds to centrosomes with full asters; weak asters correspond to centrosomes with only a few microtubules; no asters corresponds to centrosomes with no visible microtubules; and detached corresponds to centrosomes that have detached from the poles.

(E-G) Micrographs of spd-2 mutants with topi-GAL4 driving UAS-Spd-2::GFP (E), UAS-Spd-2A::GFP (F) and UAS-Spd-2B::GFP (G) stained for Tubulin (green) and Asl (magenta). 
A $\quad \square$ strong aster $\square$ weak aster

- no aster $\square$ detached

Spd-2A-B $B^{\text {chimera }}$

Spd-2B-A $A^{\text {chimera }}$

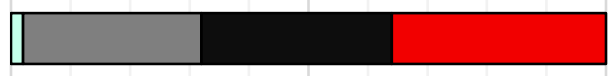

Spd-2A $A^{\Delta C \text {-tail }}$

Spd-2B+C-tail

$$
0 \%
$$$$
50 \%
$$$$
100 \%
$$

Meiotic centrosome phenotype
topi-GAL4 >

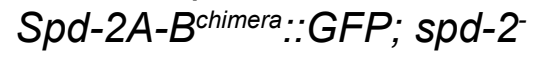

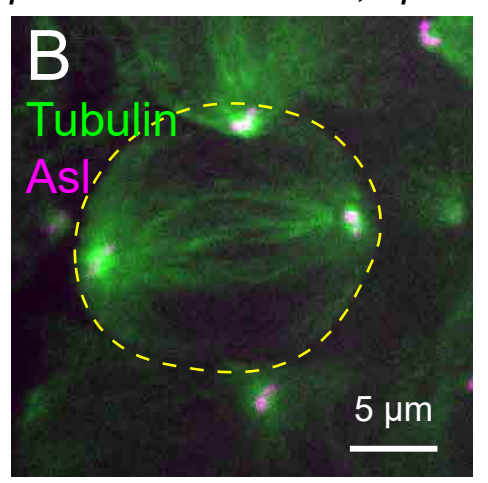

topi-GAL4 >

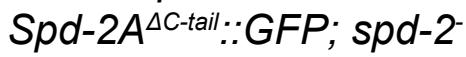

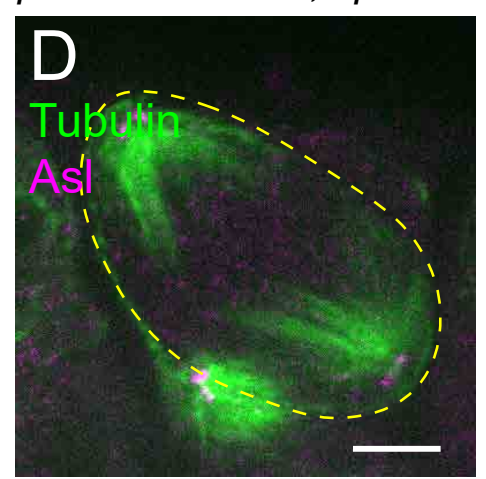

topi-GAL4 > Spd-2B-A chimera:::GFP; spd-2-

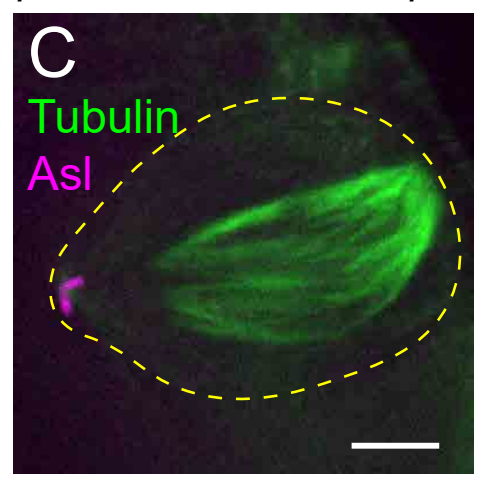

topi-GAL4 > Spd-2B+c-tail::GFP; spd-2-

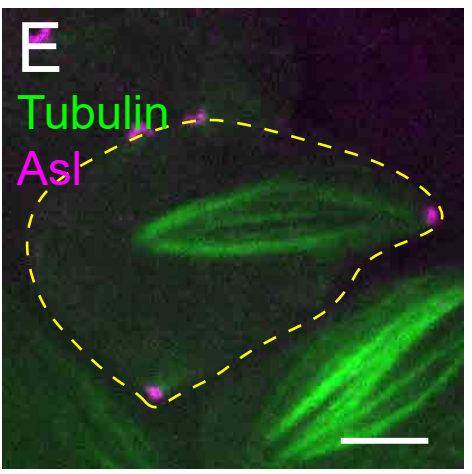




\section{Figure 9-Spd-2A C-terminal tail inhibits meiotic spindle function}

(A) Quantification of centrosome phenotypes for topi-GAL4 > chimeric transgene rescues. Strong aster corresponds to centrosomes with full asters; weak asters correspond to centrosomes with only a few microtubules; no asters corresponds to centrosomes with no visible microtubules; and detached corresponds to centrosomes that have detached from the poles.

(B-E) Micrographs of spd-2 mutants with topi-GAL4 driving UAS-Spd-2A-B ${ }^{\text {chimera }}:: G F P(B), U A S-S p d-2 B-$

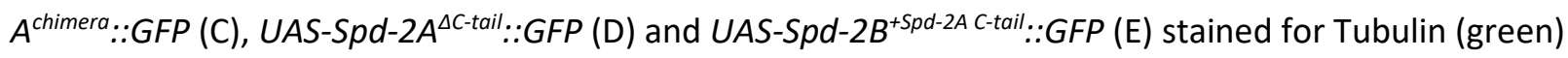
and Asl (magenta). 
A Spd-2 Two-step activation

Interphase - Inactive Tail engaged

\section{Step 1 - Priming \\ Tail disengaged}

Step 2 - Activation

Phosphorylation

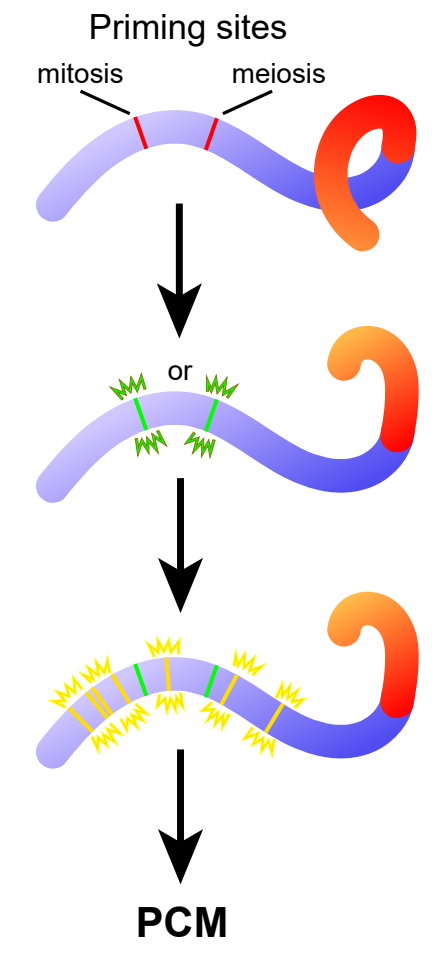

B Evolutionary events

Ancestral Spd-2

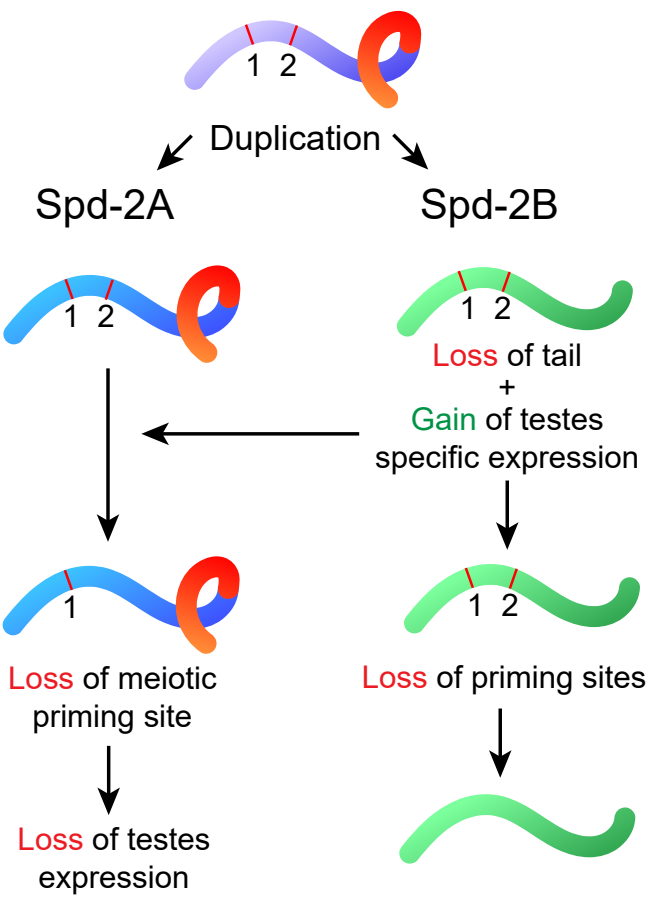

\section{Step 1}

Priming

Step 2

Activation

C

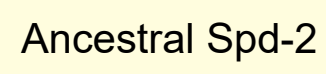

D
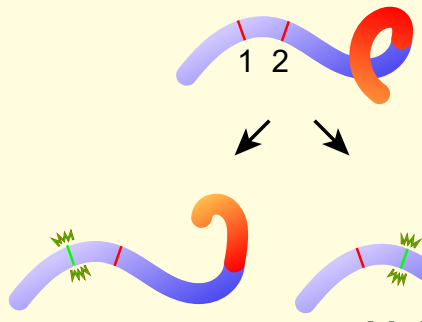

Mitosis

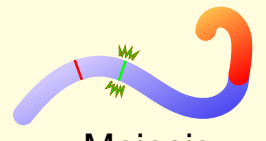

Meiosis
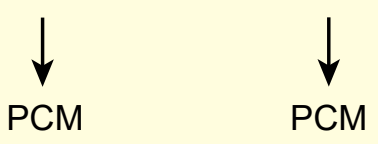

PCM

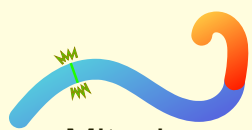

Mitosis

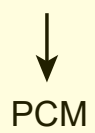

Spd-2A
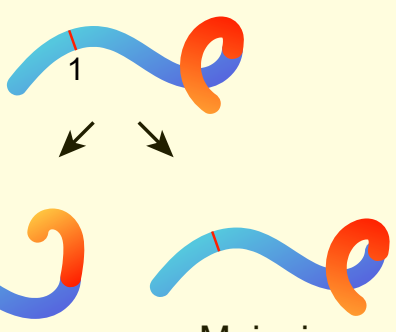

Meiosis

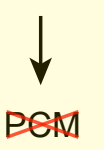

$E$

Spd-2B

(ectopic expression)

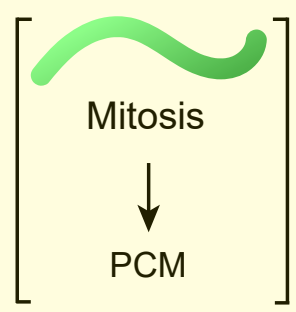

Meiosis

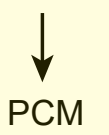

\section{Figure 10 - Hypothetical Model for Evolution of Spd-2A and Spd-2B Cell Type-Specific Specializations}




\section{Figure 10 - Hypothetical model for evolution of Spd-2A and Spd-2B cell type-specific specializations}

(A) Hypothetical 2-step model for Spd-2 activation. In interphase, Spd-2 is unphosphorylated with the Cterminal tail preventing activation. In Step 1, Spd-2 is primed for activation by relieving the C-terminal tail autoinhibition and thus allowing phosphorylation. We speculate that this priming step is achieved via distinct mitotic and meiotic regulatory sites. In Step 2, Spd-2 is phosphorylated to allow recruitment of Polo and Cnn to expand the PCM.

(B) Model of Spd-2 gene duplicate evolution. Ancestral Spd-2 is ubiquitously expressed and contains two distinct regulatory sites and the C-terminal tail. Gene duplication gave rise to Spd-2B, which gained testes-specific expression and lacked the C-terminal tail. Since Spd-2B was able to function in meiotic PCM assembly, there was no longer evolutionary pressure for Spd-2A to maintain the meiotic regulatory site or testes expression and thus they were lost. Similarly, we speculate that there would be no pressure to maintain either regulatory site in Spd-2B, and thus we predict that they would also be lost.

(C) Ancestral Spd-2 is autoinhibited via the C-terminal tail during interphase. Priming is achieved via distinct regulatory sites during mitosis or meiosis.

(D) Spd-2A, which retains the C-terminal tail, is properly primed during mitosis. However, due to loss of a hypothetical meiotic regulatory site, Spd-2A remains autoinhibited during meiosis, thus blocking phosphorylation and preventing PCM assembly.

(E) Spd-2B does not require a priming step due to loss of the C-terminal tail. Spd-2B is not expressed in somatic cells and therefore does not normally contribute to mitotic PCM, however ectopic expression leads to proper PCM assembly. Spd-2B, which is expressed in male germline cells, can freely organize PCM during meiosis because it has no C-terminal tail to block phosphorylation. 
A

Spd-2A

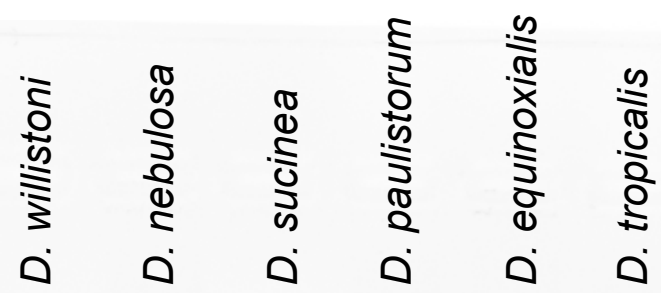

Spd-2B

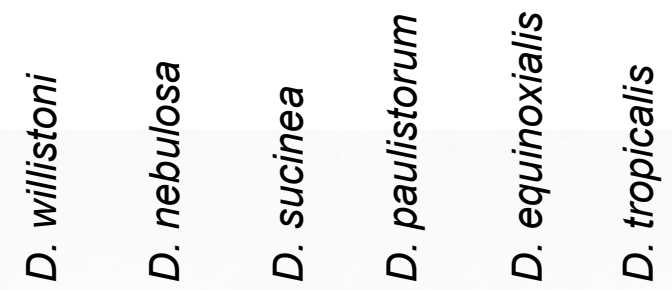

Figure S1 - Willistoni group Spd-2A and Spd-2B PCR products 
bioRxiv preprint doi: https://doi.org/10.1101/2021.10.27.466145; this version posted October 28, 2021. The copyright holder for this preprint (which was not certified by peer review) is the author/funder. All rights reserved. No reuse allowed without permission.

\section{Figure S1 - Willistoni group Spd-2A and Spd-2B PCR products}

(A) Ethidium bromide-stained agarose gel showing PCR products for Spd-2A (top) and Spd-2B (bottom) from six Willistoni group species. 
Spd-2
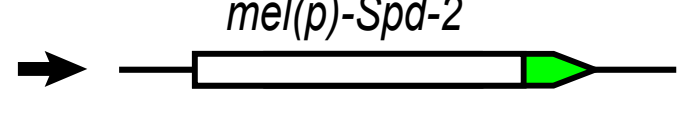

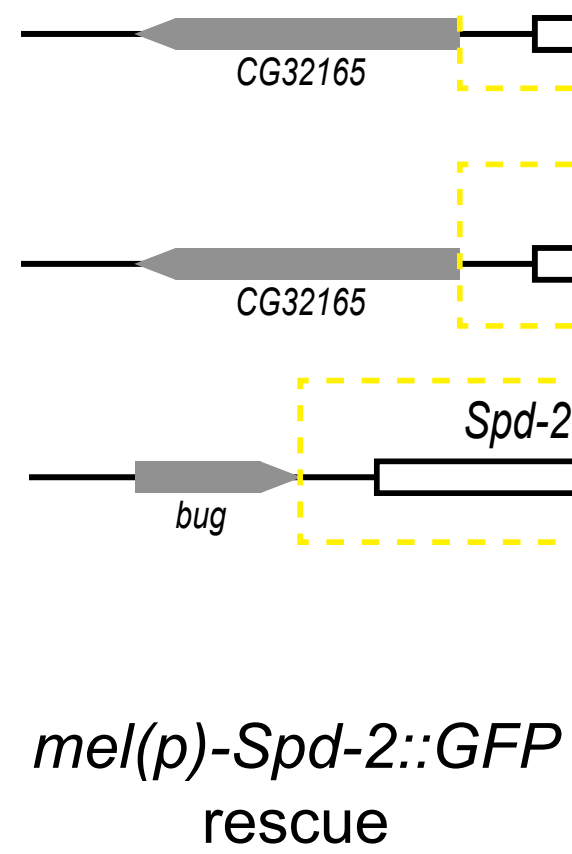

\section{Spd-2A}

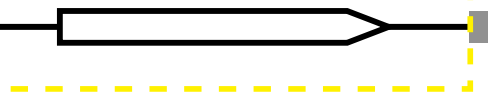

tra

Spd-2B

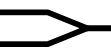

$-=$

\section{B}

GFP

\section{wil(p)-Spd-2A::GFP} rescue

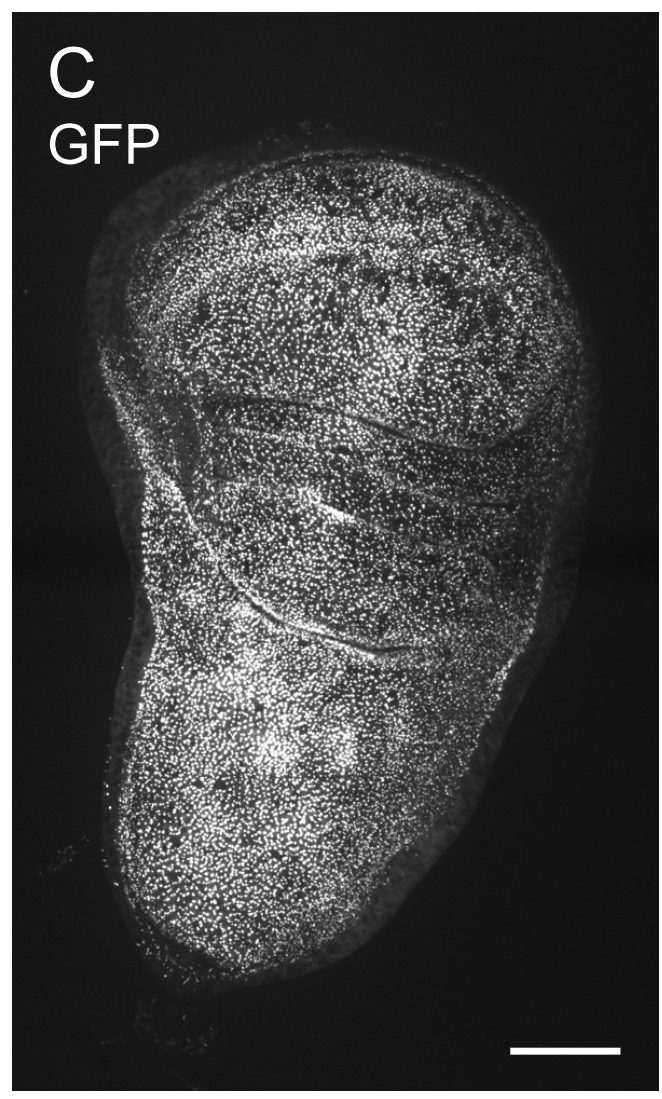

wil(p)-Spd-2B::GFP rescue

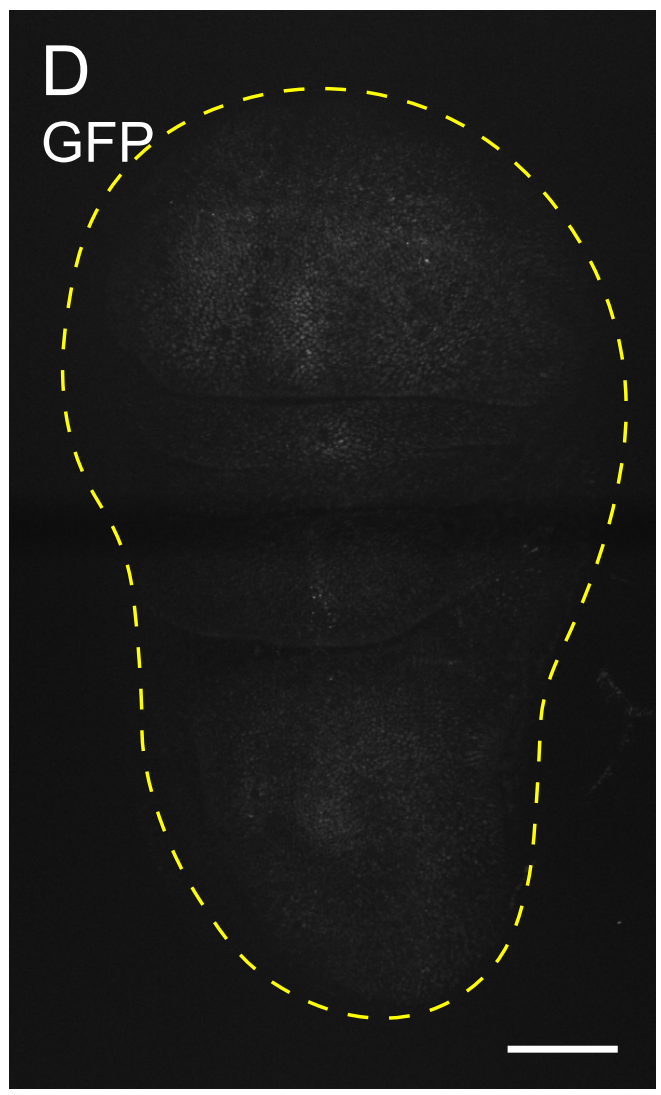

Figure $S 2$ - $S p d-2$ and $S p d-2 A$, but not $S p d-2 B$, are Expressed in Somatic Tissues 


\section{Figure $S 2-S p d-2$ and $S p d-2 A$, but not $S p d-2 B$, are expressed in somatic tissues}

(A) Diagram of native promoter transgene design. Gene regions including upstream and downstream regulatory DNA for $D$. melanogaster $S p d-2$ and $D$. willistoni $S p d-2 A$ and $S p d-2 B$ were used to generate GFP-tagged transgenes inserted into the attp40 landing site.

(B-D) Micrographs of unfixed $3^{\text {rd }}$ instar larval wing discs expressing mel(p)-Spd-2::GFP (B), wil(p)-Spd2A::GFP (C) and wil(p)-Spd-2B::GFP (D). Both Spd-2::GFP and Spd-2A::GFP are expressed in wing discs, whereas Spd-2B::GFP is undetectable. 
bioRxiv preprint doi: https://doi.org/10.1101/2021.10.27.466145; this version posted October 28, 2021. The copyright holder for this preprint (which was not certified by peer review) is the author/funder. All rights reserved. No reuse allowed without permission.

Figure S3 - Spd-2B landed in a locus with testis-biased expression

(A) Representation of the $D$. melanogaster genome region that is syntenic to the locus where $S p d-2 B$ was inserted.

(B-H) Expression profiles of genes in the Spd-2B syntenic locus, showing testis expression in red. Five out of seven genes near the Spd-2B insertion site have testis-biased expression. Expression levels were obtained from Flybase high-throughput tissue-specific expression data. 